\title{
A DISCRETE MEAN VALUE OF THE DERIVATIVE OF THE RIEMANN ZETA FUNCTION
}

\author{
NATHAN NG
}

\begin{abstract}
In this article we compute a discrete mean value of the derivative of the Riemann zeta function. This mean value will be important for several applications concerning the size of $\zeta^{\prime}(\rho)$ where $\zeta(s)$ is the Riemann zeta function and $\rho$ is a non-trivial zero of the Riemann zeta function.
\end{abstract}

\section{INTRODUCTION}

In this article we compute a discrete mean value of the Riemann zeta function, $\zeta(s)$. Throughout, $\rho=\beta+i \gamma$ will denote a non-trivial zero of the Riemann zeta function and $T$ will be a large parameter. Moreover, we define the Dirichlet polynomials

$$
X(s)=\sum_{n \leq M} \frac{x_{n}}{n^{s}} \text { and } Y(s)=\sum_{n \leq M} \frac{y_{n}}{n^{s}}
$$

where $x_{n}$ and $y_{n}$ are arbitrary real sequences and $M=T^{\theta}$ with $0<\theta<1 / 2$. We shall evaluate the following mean value:

$$
S=\sum_{0<\gamma<T} \zeta^{\prime}(\rho) X(\rho) Y(1-\rho) .
$$

However, our main purpose for evaluating $S$ is to employ it for an application concerning large values of $\zeta^{\prime}(\rho)$. In an accompanying paper [13] we prove the following results:

Theorem 1.1. Assume the Riemann Hypothesis. For each $A>0$, we have

$$
\left|\zeta^{\prime}(\rho)\right| \gg(\log |\gamma|)^{A}
$$

for infinitely many $\rho=\frac{1}{2}+i \gamma$.

In order to strengthen this result we will require an additional assumption concerning the location of zeros of Dirichlet $L$-functions.

\section{Large zero-free region conjecture.}

There exists a constant $c_{0}>0$ sufficiently large such that for each $q \geq 1$ and each character $\chi$ modulo $q$ the Dirichlet $L$-function $L(s, \chi)$ does not vanish in the region

$$
\sigma \geq 1-\frac{c_{0}}{\log \log (q(|t|+4))}
$$

November 10, 2018. Mathematics Subject Classification (2000). 11M26. This research was funded in part by NSERC and NSF FRG grant DMS 0244660. 
where $s=\sigma+i t$.

The value of $c_{0}$ required may be calculated and $c_{0}=100$ suffices but is not minimal. We note that this large zero-free region conjecture is significantly weaker than the Generalized Riemann Hypothesis which asserts that all non-trivial zeros of each Dirichlet $L$-function lie on the one-half line. On the other hand, this zero-free region is still much larger than what has currently been proven. For example, this conjecture rules out the existence of Siegel zeros.

Theorem 1.2. Assume the Riemann hypothesis and the large zero-free conjecture for Dirichlet L-functions. There exists a constant $c_{2}>0$ such that

$$
\left|\zeta^{\prime}(\rho)\right| \gg \exp \left(c_{2} \sqrt{\frac{\log |\gamma|}{\log \log |\gamma|}}\right)
$$

for infinitely many $\rho=\frac{1}{2}+i \gamma$.

The methods employed to prove Theorems 1.1 and 1.2 are based on Soundararajan's [17] resonance method. This method has proven to be successful in determining extreme values of $L$-functions and character sums. In the article [13] we also succeed in exhibiting small values of $\left|\zeta^{\prime}(\rho)\right|$ too.

We also note that an asymptotic evaluation of $S$ has other important applications. Soundararajan has informed me that he can prove under the assumption of the Riemann hypothesis that

$$
\sum_{0<\gamma<T}\left|\zeta^{\prime}(\rho)\right|^{2 k} \gg_{k} T(\log T)^{(k+1)^{2}} .
$$

This proof requires our formula for $S$ and follows the lower bound method of Rudnick and Soundararajan [14], [15]. We observe that this is stronger than Theorem 1.1.

Our evaluation of $S$ will be split in two cases depending on the properties of the coefficients $x_{n}$ and $y_{n}$. The cases we shall consider are:

Case 1. The divisor case. The coefficients shall satisfy the bounds

$$
\left|x_{n}\right|,\left|y_{n}\right| \leq \tau_{r}(n)(\log T)^{C}
$$

for $r \in \mathbb{N}$ and $C \geq 0$ where $\tau_{r}(n)$ is the $r$-th divisor function. We shall also assume

$$
\left|x_{m n}\right| \ll\left|x_{m}\right|\left|x_{n}\right| \text { and }\left|y_{m n}\right| \ll\left|y_{m}\right|\left|y_{n}\right| \text {. }
$$

Case 2. The resonator case. In this case we will take $x_{n}=y_{n}=f(n)$ where $f$ is a multiplicative function supported on the squarefree integers. For a prime $p$ we define

$$
f(p)= \begin{cases}\frac{L}{\log p} & \text { if } L^{2} \leq p \leq \exp \left((\log L)^{2}\right) \\ 0 & \text { else }\end{cases}
$$


where $L=\sqrt{\log M \log \log M}$.

The resonator coefficients have recently been employed by Soundararajan [17] and they arose in a certain optimization problem related to finding extreme values of $\zeta(1 / 2+i t)$. We shall refer throughout this article to case one as the divisor case and case two as the resonator case. Our evaluation of $S$ in the divisor case will be unconditional whereas the evaluation of $S$ in the resonator case will depend on the (as yet) unproven large zero-free region conjecture.

We now state our result for $S$. We let $c_{j}$ for $j=1,2,3, \ldots$ denote positive constants.

Theorem 1.3. (i) If $x_{n}, y_{n}$ satisfy (2), (3) then

$$
\begin{aligned}
S & =\frac{T}{2 \pi} \sum_{n u \leq M} \frac{x_{u} y_{n u} r_{0}(n)}{n u}-\frac{T}{4 \pi} \sum_{n u \leq M} \frac{y_{u} x_{u n}}{n u} R_{2}\left(\log \left(\frac{T}{2 \pi n}\right)\right) \\
& +\frac{T}{2 \pi} \sum_{\substack{a, b \leq M \\
(a, b)=1}} \frac{r_{1}(a, b)}{a b} \sum_{g \leq \min \left(\frac{M}{a}, \frac{M}{b}\right)} \frac{y_{a g} x_{b g}}{g}+\tilde{\mathcal{E}}
\end{aligned}
$$

where for $0<\theta<1 / 2$ we have for any $A^{\prime}>0$

$$
\tilde{\mathcal{E}} \ll_{A^{\prime}} T(\log T)^{-A^{\prime}}+T^{\frac{3}{4}+\frac{\theta}{2}+\epsilon} .
$$

The other quantities are defined as follows:

$$
\begin{gathered}
r_{0}(n)=P_{2}\left(\log \left(\frac{T}{2 \pi}\right)\right)-2 P_{1}\left(\log \left(\frac{T}{2 \pi}\right)\right)(\log n)+(\Lambda * \log )(n), \\
r_{1}(a, b)=\frac{1}{2} \Lambda_{2}(a)-R_{1}\left(\log \left(\frac{T}{2 \pi b}\right)\right) \Lambda(a)-\tilde{R}_{1}\left(\log \left(\frac{T}{2 \pi b}\right)\right) \alpha_{1}(a)-\alpha_{2}(a),
\end{gathered}
$$

$P_{1}, P_{2}, R_{1}, \tilde{R}_{1}, R_{2}$ are monic polynomials of degrees $1,2,1,1,2$ respectively and $\alpha_{1}, \alpha_{2}$ are arithmetic functions. In fact, $\alpha_{1}$ is supported on prime powers, $\alpha_{2}$ is supported on integers $n$ such that $\omega(n) \leq 2$. More precisely, $\alpha_{1}\left(p^{\alpha}\right)=\frac{\log p}{p-1}, \alpha_{2}\left(p^{\alpha}\right)=-\frac{(\alpha+1)(\log p)^{2}}{p-1}+\frac{D \log p}{p-1}-\frac{\log p}{(p-1)^{2}}$ for some $D \in \mathbb{R}$, and $\alpha_{2}\left(p^{\alpha} q^{\beta}\right)=-(\log p)(\log q)\left(\frac{1}{p-1}+\frac{1}{q-1}\right)$ for $\alpha, \beta \in \mathbb{N}$.

(ii) Assume the large zero-free region conjecture. If $x_{n}=y_{n}=f(n)$ where $f$ is defined by (4) then there exists a $c_{1}>0$ such that (5) remains true with

$$
\tilde{\mathcal{E}} \ll T \exp \left(-\frac{c_{1} \log T}{\log \log T}\right)+T^{\frac{3}{4}+\frac{3 \theta}{2}+\epsilon}
$$

for $0<\theta<1 / 6$.

Remarks. 1. It is possible to obtain an intermediate result to Theorems 1.1 and 1.2. In fact, one can show that if the Riemann hypothesis is true and there are no Siegel zeros then there exists a $c_{3}>0$ such that

$$
\left|\zeta^{\prime}(\rho)\right| \gg \exp \left(c_{3}(\log |\gamma|)^{1 / 4}\right)
$$


infinitely often. The proof of this result rests on deriving Theorem 1.3 for the sequences $x_{n}=y_{n}=g(n)$ where $g$ is multiplicative and supported on squarefree integers. On primes it is defined by

$$
g(p)= \begin{cases}\frac{c(\log M)^{1 / 4} \log \log M}{\log p} & \text { if } A \leq p \leq B \\ 0 & \text { else }\end{cases}
$$

for some $c>0$. The evaluation of $S$ in this case is very similar to the two other cases worked out in the article. However, due to the length of this article we have decided not to present this case.

2. Various mean values involving $\zeta^{\prime}(\rho)$ have been explored in several previous articles. (See [2], 3], [5], [7, 10].) Discrete moments of $\zeta^{\prime}(\rho)$ have number theoretic applications to simple zeros of the zeta function [3] and to the distribution of the summatory function of the Möbius function [11]. More generally, moments of $X(\rho+\alpha)$ for a Dirichlet polynomial $X(s)$ and $\alpha \in \mathbb{C}$ have applications to extreme gaps between the zeros of the zeta function (see [1, [12]).

3. The argument for this theorem is based on an argument of Conrey, Ghosh, and Gonek [3] for evaluating the sums

$$
S_{1}=\sum_{0<\gamma<T} \zeta^{\prime}(\rho) X(\rho) \text { and } S_{2}=\sum_{0<\gamma<T} \zeta^{\prime}(\rho) \zeta^{\prime}(1-\rho) X(\rho) X(1-\rho)
$$

where $x_{n}=\mu(n) P\left(\frac{\log (M / n)}{\log M}\right)$ and $P$ is a polynomial. The evaluation of $S_{2}$ requires the assumption of the generalized Lindelöf hypothesis whereas $S_{1}$ may be computed unconditionally. Oddly, this point is never stressed in [3]. In a future article, we shall evaluate the above sums with arbitrary coefficients by the methods of this article.

4. The proof of the bound for $\tilde{\mathcal{E}}$ in Theorem 1 is obtained by an argument which is very similar to the proof of the Bombieri-Vinogradov theorem. Recall that it asserts that for each $A>0$

$$
\sum_{q \leq M} \max _{(a, q)=1} \max _{y \leq T}\left|\psi(y ; a, q)-\frac{y}{\phi(q)}\right| \ll T(\log T)^{-A}+T^{1 / 2} M(\log T M)^{6} .
$$

Improving our value of $M$ beyond $\sqrt{T}$ in Theorem 1.3 lies as deep as improving the Bombieri-Vinogradov theorem for $M$ larger than $\sqrt{T}$.

5. In this article we must impose some conditions on the location of zeros of Dirichlet $L$-functions. More precisely, we assume the large zero-free region conjecture for Dirichlet $L$-functions in order to evaluate $S$ in the resonator case. The central reason for applying this conjecture is that the coefficients $f(n)$ satisfy $\sum_{n \leq M} f(n)^{2} \sim M \exp \left(\frac{c \log T}{\log \log T}\right)$. Now the general setup for evaluating $S_{1}$ in the article of [3] is to use an argument similar to proving the Bombieri-Vinogradov theorem. However, this type of argument provides a 
savings of $(\log T)^{-A}$ for any $A$ from the main term. As the resonator coefficients $x_{n}=y_{n}=f(n)$ become very large in mean square we will be unable to obtain an asymptotic formula with only a savings of a power of a logarithm. The central reason for applying the large zero free region conjecture is that it allows us to have a savings of $\exp \left(-\frac{c \log T}{\log \log T}\right)$ for a large enough $c$ which balances the large average size of the resonator coefficients. On the other hand, for the evaluation of $S_{2}$ in [3] the coefficients $x_{n}, y_{n}$ are bounded in size. In that case the Generalized Lindelöf hypothesis is invoked in order to bound a sixth integral moment of $L(s, \chi)$ on average on the critical line and has nothing to do with the size of the coefficients $x_{n}, y_{n}$ as in our case.

Acknowledgements. I would like to thank K. Soundararajan for suggesting this problem. Also thanks to H. Kadiri for a useful question.

\section{Notation}

Throughout out this article we shall denote a series of positive constants by $c_{j}$ and $C_{j}$ for $j=1,2, \ldots$. We remark that some of the constants $C_{j}$ will depend on the numbers $r$ and $C$ given in (2). For $T$ large we define $\mathscr{L}=\log (T)$. We shall also consider arbitrary sequences $\mathbf{x}=\left\{x_{n}\right\}$ supported on the interval $[1, M]$. We shall employ the notation

$$
\|\mathbf{x}\|_{\infty}=\max _{n \leq M}\left|x_{n}\right| \text { and }\|\mathbf{x}\|_{p}=\left(\sum_{n \leq M}\left|x_{n}\right|^{p}\right)^{1 / p} .
$$

We shall use Vinogradov's notation $f(x) \ll g(x)$ to mean there exists a $C>0$ such that $|f(x)| \leq C g(x)$ for all $x$ sufficiently large. We denote $f(x)=O(g(x))$ to mean the same thing.

In addition, we will encounter a host of familiar arithmetic functions. Let $\omega(n)$ denote the number of distinct prime factors of $n$. For $r>0$ we define $\tau_{r}(n)$, the $r$-th divisor function, to be the coefficient of $n^{-s}$ in the Dirichlet series $\zeta^{r}(s)$. If $r=2$ we write $\tau(n)=\tau_{2}(n)$. Similarly $\Lambda(n)$ is the coefficient of $n^{-s}$ in the Dirichlet series of $-\zeta^{\prime}(s) / \zeta(s)$. This yields the expression $\Lambda(n)=\sum_{d \mid n} \mu(d) \log \frac{n}{d}$. Moreover, we have its generalization $\Lambda_{k}=\mu * \log ^{k}$. An equivalent definition is that $\Lambda_{k}(n)$ is the coefficient of $n^{-s}$ in the Dirichlet series $(-1)^{k} \zeta^{(k)}(s) / \zeta(s)$. Furthermore, $\Lambda_{k}(n)=0$ is supported on those integers with at most $k$ prime factors. We also define $j(n)=\prod_{p \mid n}\left(1+10 p^{-1 / 2}\right)$.

\section{The Dirichlet polynomial COEFFiCIENTS $x_{n}$}

We record some properties of the coefficients that will be employed throughout the article:

\section{Properties of the divisor coefficients.}

Let $x_{n}$ and $y_{n}$ satisfy (21) and (3). We have the standard estimates:

$$
\begin{aligned}
& \|\mathbf{x}\|_{\infty},\|\mathbf{y}\|_{\infty} \ll T^{\epsilon}, \\
& \left\|\frac{x_{n}}{n}\right\|_{1},\left\|\frac{y_{n}}{n}\right\|_{1},\left\|\frac{x_{n}^{2}}{n}\right\|_{1},\left\|\frac{y_{n}^{2}}{n}\right\|_{1},\left\|\frac{x_{n}\left(\tau_{k} * y\right)(n)}{n}\right\| \ll \mathscr{L}^{C^{\prime}}
\end{aligned}
$$


for some $C^{\prime}>0$ and $k \in \mathbb{N}$. We remark that the above bounds remain true when the above sequences are multiplied by $j(n)$.

\section{Properties of the resonator coefficients.}

Let $f$ be defined by (44). We have the following estimates:

$$
\begin{gathered}
f(m n) \ll f(m) f(n) \\
\|f\|_{\infty} \leq M^{1 / 2+\epsilon} \text { for } M \text { sufficiently large }, \\
\|f\|_{1} \ll M \exp \left((1+o(1)) \sqrt{\frac{\log M}{\log \log M}}\right) \\
\left\|f^{2}\right\|_{1} \ll M \exp \left((0.5+o(1)) \frac{\log M}{\log \log M}\right) \\
\sum_{n \leq M} \frac{f(n)}{n} \ll \exp \left((1+o(1)) \sqrt{\frac{\log M}{\log \log M}}\right) \\
\sum_{n \leq M} \frac{f(n)^{2}}{n} \ll \exp \left((0.5+o(1)) \frac{\log M}{\log \log M}\right) \\
\sum_{n \leq M} \frac{j(n)\left(\tau_{r} * f\right)(n) f(n)}{n} \ll \exp \left((0.5+o(1)) \frac{\log M}{\log \log M}\right) \\
\sum_{n \leq T} \frac{(\tau * f)(n)^{2}}{n}, \sum_{n \leq T} \frac{(f * f)(n)^{2}}{n} \ll T^{\epsilon} .
\end{gathered}
$$

We now give an indication of how to prove (9). The proofs of the other inequalities are similar. We denote $\Sigma$ the sum to be estimated.

$$
\Sigma \leq \sum_{n=1}^{\infty} \frac{j(n)\left(\tau_{r} * f\right)(n) f(n)}{n}=\prod_{p}\left(1+\frac{j(p)\left(\tau_{r} * f\right)(p) f(p)}{p}\right) .
$$

Since $j(p)=1+O\left(p^{-1 / 2}\right), f(p)=\frac{L}{\log p},\left(\tau_{r} * f\right)(p)=\frac{L}{\log p}+r$ we obtain

$$
\log (\Sigma) \leq \sum_{L^{2} \leq p \leq \exp \left((\log L)^{2}\right)}\left(\frac{L^{2}}{p(\log p)}+O\left(\frac{L}{p(\log p)}+\frac{L^{2}}{p^{3 / 2}}\right)\right) .
$$

By the prime number theorem

$$
\sum_{L^{2} \leq p \leq \exp \left((\log L)^{2}\right)} \frac{1}{p(\log p)^{2}}=\frac{1}{8(\log L)^{2}}(1+o(1))
$$

and thus $\Sigma \leq \exp \left(\frac{\log M}{2 \log \log M}(1+o(1))\right)$.

\section{Preliminary manipulations of $S$ and proof of Theorem 1.3}

Proof. We commence with our evaluation of $S$. We start with some initial manipulations. Recall that our goal is to evaluate

$$
S=\sum_{0<\gamma<T} \zeta^{\prime}(\rho) X(\rho) Y(1-\rho)
$$


The functional equation for the Riemann zeta function is $\zeta(s)=\chi(s) \zeta(1-s)$, where

$$
\chi(1-s)=\chi(s)^{-1}=2(2 \pi)^{-s} \Gamma(s) \cos (\pi s / 2) .
$$

Differentiating the functional equation,

$$
\zeta^{\prime}(s)=-\chi(s)\left(\zeta^{\prime}(1-s)-\frac{\chi^{\prime}}{\chi}(s) \zeta(1-s)\right)
$$

From this last equation it follows that

$$
\begin{aligned}
S & =-\sum_{0<\gamma<T} \chi(\rho) \zeta^{\prime}(1-\rho) X(\rho) Y(1-\rho) \\
& =\frac{1}{2 \pi i} \int_{\mathcal{C}} \frac{\zeta^{\prime}}{\zeta}(1-s) \chi(s) \zeta^{\prime}(1-s) X(s) Y(1-s) d s
\end{aligned}
$$

where $\mathcal{C}$ is the positively oriented rectangle with vertices at $1-\kappa+i, \kappa+$ $i, \kappa+i T$, and $1-\kappa+i T$, and $\kappa=1+\mathscr{L}^{-1}$. Moreover, we choose $T$ so that the distance from $T$ to the nearest zero is $\gg \mathscr{L}^{-1}$. The bottom edge of this contour is clearly $O(1)$. On the top edge we have the standard bounds

$$
\begin{aligned}
\chi(s) & \ll T^{1 / 2-\sigma}, \\
\zeta^{\prime}(1-s) & \ll T^{\sigma / 2+\epsilon}, \\
X(s) & \ll M^{1-\sigma}\left\|\frac{x_{n}}{n}\right\|_{1}, \\
Y(1-s) & \ll M^{\sigma}\left\|\frac{y_{n}}{n}\right\|_{1}, \\
\frac{\zeta^{\prime}}{\zeta}(1-s) & \ll \mathscr{L}^{2} .
\end{aligned}
$$

Note that the last bound only holds for $s=\sigma+i t$ as long as $|t-\gamma| \gg \mathscr{L}^{-1}$ for all imaginary ordinates $\gamma$. Combining these bounds shows that the top edge of the contour is bounded by $M T^{1 / 2+\epsilon}$. Next note that

$$
\frac{\zeta^{\prime}}{\zeta}(1-s)=\frac{\chi^{\prime}}{\chi}(s)-\frac{\zeta^{\prime}}{\zeta}(s)
$$

and

$$
\chi(s) \zeta^{\prime}(1-s)=-\zeta^{\prime}(s)+\frac{\chi^{\prime}}{\chi}(s) \zeta(s)
$$

imply the right-hand side of the integral is

$$
S_{R}=\frac{1}{2 \pi i} \int_{\kappa+i}^{\kappa+i T}\left(\frac{\chi^{\prime}}{\chi}(s)^{2} \zeta(s)-2 \frac{\chi^{\prime}}{\chi}(s) \zeta^{\prime}(s)+\frac{\zeta^{\prime}}{\zeta}(s) \zeta^{\prime}(s)\right) X(s) Y(1-s) d s .
$$

The left-hand side is

$$
S_{L}=\frac{1}{2 \pi i} \int_{1-\kappa+i T}^{1-\kappa+i} \frac{\zeta^{\prime}}{\zeta}(1-s) \chi(s) \zeta^{\prime}(1-s) X(s) Y(1-s) d s .
$$

By the variable change $s \rightarrow 1-s$ the left side equals $-\bar{I}_{L}$ where

$$
I_{L}=\frac{1}{2 \pi i} \int_{\kappa+i}^{\kappa+i T} \chi(1-s) \frac{\zeta^{\prime}}{\zeta}(s) \zeta^{\prime}(s) X(s) Y(1-s) d s
$$


We have now demonstrated that

$$
S=S_{R}-\bar{I}_{L}+O\left(M T^{1 / 2+\epsilon}\right)
$$

with $S_{R}$ and $I_{L}$ defined as above. We now set up the evaluation of $I_{L}$. We begin by writing

$$
\frac{\zeta^{\prime}}{\zeta}(s) \zeta^{\prime}(s) A(s)=\sum_{m=1}^{\infty} a(m) m^{-s}
$$

where

$$
a(m)=\sum_{u v w=m} \Lambda(u) \log (v) x_{w}
$$

It thus follows

$$
I_{L}=\sum_{k \leq M} \frac{y_{k}}{k} \sum_{m=1}^{\infty} a(m) \frac{1}{2 \pi i} \int_{\kappa+i}^{\kappa+i T} \chi(1-s)(m / k)^{-s} d s
$$

and we invoke

Lemma 4.1. Let $r, \kappa_{0}>0$ we have

$$
\frac{1}{2 \pi i} \int_{\kappa+i}^{\kappa+i T} \chi(1-s) r^{-s} d s=\delta(r) e(-r)+E(r, c) r^{-\kappa}
$$

uniformly for $\kappa_{0} \leq \kappa \leq 2$ where $\delta(r)=1$ if $r \leq T / 2 \pi$ and $\delta(r)=0$ otherwise. Moreoever,

$$
E(r, \kappa) \ll T^{\kappa-1 / 2}+\frac{T^{\kappa+1 / 2}}{|T-2 \pi r|+T^{1 / 2}} .
$$

This result follows from Lemma 2 of [7]. Applying Lemma 4.1 yields $I_{L}=$ $\mathcal{M}+T^{1 / 2} \mathcal{E}_{1}^{\prime}+T^{3 / 2} \mathcal{E}_{2}^{\prime}$ where

$$
\begin{gathered}
\mathcal{M}=\sum_{k \leq M} \frac{y_{k}}{k} \sum_{m \leq \frac{k T}{2 \pi}} a(m) e\left(-\frac{m}{k}\right), \\
\mathcal{E}_{1}^{\prime}=T^{1 / 2} \sum_{k \leq M}\left|y_{k}\right| \sum_{m=1}^{\infty} \frac{|a(m)|}{m^{\kappa}}, \\
\mathcal{E}_{2}^{\prime}=T^{3 / 2} \sum_{k \leq M}\left|y_{k}\right| \sum_{m=1}^{\infty} \frac{|a(m)|}{m^{\kappa}}\left(|T-2 \pi m / n|+T^{1 / 2}\right)^{-1} .
\end{gathered}
$$

Note that

$$
\mathcal{E}_{1}^{\prime} \ll\|\mathbf{y}\|_{1} \sum_{m \leq M} \frac{\left|x_{m}\right|}{m} \frac{\zeta^{\prime}}{\zeta}(\kappa) \zeta^{\prime}(\kappa) \ll \mathscr{L}^{3}\|\mathbf{y}\|_{1}\left\|\frac{x_{m}}{m}\right\|_{1} .
$$

We next consider $\mathcal{E}_{2}^{\prime}$. We split this into the cases: $(i)|T-2 \pi m / n|>T / 2$, (ii) $\sqrt{T} \leq|T-2 \pi m / n| \leq T / 2$, and $($ iii $)|T-2 \pi m / n| \leq \sqrt{T}$. In case $(i)$, $g_{m, n}(T) \ll T^{-1}$ and we have

$$
\mathcal{E}_{21}^{\prime} \ll T^{-1}|| \mathbf{y}\left\|_{1} \sum_{m=1}^{\infty} \frac{|a(m)|}{m^{\kappa}} \ll T^{-1} \mathscr{L}^{3}\right\| \mathbf{y}\left\|_{1} \mid\right\| \frac{x_{m}}{m} \|_{1} .
$$


In case $($ ii) we begin by assuming without loss of generality that $\sqrt{T} \leq$ $2 \pi m / n-T \leq T / 2$. We divide this into $\ll \log T$ intervals of the shape $T+P<2 \pi m / n<T+2 P$ with $\sqrt{T} \ll P \ll T$. We denote the interval $I=\left[\frac{n T}{2 \pi}+\frac{n P}{2 \pi}, \frac{n T}{2 \pi}+\frac{2 n P}{2 \pi}\right]$. Note that $|a(m)| \leq\|\mathbf{x}\|_{\infty} \tau(m) \log ^{2} m$ and hence

$$
\begin{aligned}
\mathcal{E}_{22}^{\prime} & \ll\|\mathbf{x}\|_{\infty} \sum_{P} \sum_{n \leq M}\left|y_{n}\right| \sum_{m \in I} \frac{\tau(m) \log ^{2} m}{m P} \\
& \ll T^{-1}|| \mathbf{x} \|_{\infty} \sum_{P} \sum_{n \leq M} \frac{\left|y_{n}\right|}{n P} \sum_{m \in I} \tau(m) \log ^{2} m \\
& \ll T^{-1}\|\mathbf{x}\|_{\infty} \sum_{P} \sum_{n \leq M} \frac{\left|y_{n}\right|}{n P}(n P) \mathscr{L}^{3} \ll T^{-1} \mathscr{L}^{4}\|\mathbf{x}\|_{\infty}\|\mathbf{y}\|_{1} .
\end{aligned}
$$

In the second inequality above we apply an estimate for the divisor sum in short intervals. For a precise statement, see Lemma 6.3 which occurs later in the article. In the last case we have $|T-2 \pi m / n| \leq \sqrt{T}$ and $g_{m, n}(T) \ll T^{-1 / 2}$. Put $J=\left[\frac{n}{2 \pi}(T-\sqrt{T}), \frac{n}{2 \pi}(T+\sqrt{T})\right]$. We now have

$$
\begin{aligned}
\mathcal{E}_{23}^{\prime} & \ll T^{-1 / 2}|| \mathbf{x}\left\|_{\infty} \sum_{n \leq M}\left|y_{n}\right| \sum_{m \in J} \frac{\tau(m) \log ^{2} m}{m} \ll T^{-3 / 2}\right\| \mathbf{x} \|_{\infty} \sum_{n \leq M} \frac{\left|y_{n}\right|}{n}(n \sqrt{T}) \mathscr{L}^{3} \\
& \ll T^{-1} \mathscr{L}^{3}\|\mathbf{x}\|_{\infty}\|\mathbf{y}\|_{1} .
\end{aligned}
$$

Combining our estimates yields $T^{1 / 2} \mathcal{E}_{1}^{\prime}+T^{3 / 2} \mathcal{E}_{2}^{\prime}$ is bounded by

$$
T^{1 / 2}\left(\mathscr{L}^{3}\|\mathbf{y}\|_{1}\left\|x_{m} / m\right\|_{1}+\mathscr{L}^{4}\|\mathbf{x}\|_{\infty}\|\mathbf{y}\|_{1}\right) \ll T^{1 / 2} \mathscr{L}^{4}\|\mathbf{y}\|_{1}\|\mathbf{x}\|_{\infty}
$$

and hence

$$
S=S_{R}-\overline{\mathcal{M}}+O\left(T^{1 / 2} \mathscr{L}^{4}\|\mathbf{y}\|_{1}\|\mathbf{x}\|_{\infty}\right)
$$

where $S_{R}$ and $\mathcal{M}$ are given by (11) and (12) respectively. We now simplify our expression (12) for $\mathcal{M}$. The first step will be to express the additive character $e(-m / k)$ in terms of multiplicative characters. In order to do this we write $m / k=m^{\prime} / k^{\prime}$ with $\left(m^{\prime}, k^{\prime}\right)=1$. We have the well-known identity

$$
e\left(-\frac{m}{k}\right)=e\left(-\frac{m^{\prime}}{k^{\prime}}\right)=\frac{1}{\phi\left(k^{\prime}\right)} \sum_{\chi \bmod k^{\prime}} \tau(\bar{\chi}) \chi\left(-m^{\prime}\right)
$$

where for a character $\chi$ modulo $k^{\prime}, \tau(\chi)=\sum_{a=1}^{k^{\prime}} \chi(a) e\left(a / k^{\prime}\right)$ is the usual Gauss sum. Now note that $\tau\left(\chi_{0}\right)=\mu\left(k^{\prime}\right)$, where $\chi_{0}$ is the principal character modulo $k^{\prime}$. Hence

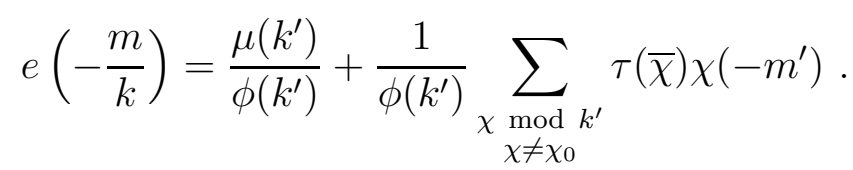

The basic idea is that when we insert the expression (16) back in (12) that $\mu\left(k^{\prime}\right) / \phi\left(k^{\prime}\right)$ term will account for the main term of $\mathcal{M}$ and the sum over non-principal characters modulo $k^{\prime}$ will be an error term. Before commencing with this strategy, we must first convert the above sum to a sum over primitive characters. This is since we shall invoke an analytic version of 
the large sieve inequality involving only primitive characters. If a character $\chi$ modulo $k^{\prime}$ is induced by the primitive character $\psi$ modulo $q$ then we have $\tau(\chi)=\mu\left(k^{\prime} / q\right) \psi\left(k^{\prime} / q\right) \tau(\psi)$ (see [4, p. 67]). We shall use the notation $\sum_{\psi \bmod q}^{*}$ to denote summation over primitive characters modulo $q$. Therefore

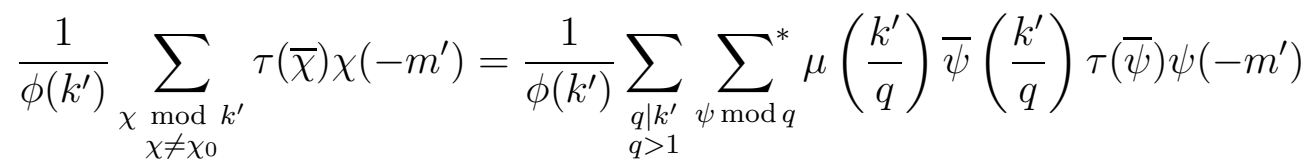

since $\left(m^{\prime}, k^{\prime}\right)=1$ implies $\left(m^{\prime}, q\right)=1$ and thus $\chi\left(-m^{\prime}\right)=\psi\left(-m^{\prime}\right)$. The next step is to rewrite this formula in terms of $m$ and $k$. Let $g=(m, k)$. By the Möbius inversion formula, we have

$$
f\left(m^{\prime}, k^{\prime}\right)=f\left(\frac{m}{g}, \frac{k}{g}\right)=\sum_{d \mid g} \sum_{e \mid d} \mu\left(\frac{d}{e}\right) f\left(\frac{m}{e}, \frac{k}{e}\right)
$$

for any function $f$. Moreover, note that the condition $d \mid g$ is equivalent to $d|m, d| k$. Thus we derive

$$
\begin{aligned}
& \frac{1}{\phi\left(k^{\prime}\right)} \sum_{\substack{\bmod k^{\prime} \\
\chi \neq \chi_{0}}} \tau(\bar{\chi}) \chi\left(-m^{\prime}\right) \\
& =\sum_{\substack{d|m \\
d| k}} \sum_{e \mid d} \frac{\mu(d / e)}{\phi(k / e)} \sum_{\substack{q \mid k / e \\
q>1}} \sum_{\psi \bmod q}^{*} \mu\left(\frac{k}{e q}\right) \bar{\psi}\left(\frac{k}{e q}\right) \tau(\bar{\psi}) \psi\left(-\frac{m}{e}\right) \\
& =\sum_{\substack{q \mid k \\
q>1}} \sum_{\bmod q}^{*} \tau(\bar{\psi}) \sum_{\substack{d|m \\
d| k}} \sum_{\substack{e|d \\
e| k / q}} \frac{\mu(d / e)}{\phi(k / e)} \bar{\psi}\left(-\frac{k}{e q}\right) \psi\left(\frac{m}{e}\right) \mu\left(\frac{k}{e q}\right) \\
& =\sum_{\substack{q \mid k \\
q>1}} \sum_{\psi \bmod q}^{*} \tau(\bar{\psi}) \sum_{\substack{d|m \\
d| k}} \psi\left(\frac{m}{d}\right) \delta(q, k, d, \psi)
\end{aligned}
$$

where

$$
\delta(q, k, d, \psi)=\sum_{\substack{e|d \\ e| k / q}} \frac{\mu(d / e)}{\phi(k / e)} \bar{\psi}\left(-\frac{k}{e q}\right) \psi\left(\frac{d}{e}\right) \mu\left(\frac{k}{e q}\right) .
$$

By (12), (16), and (17) we have now shown that $\mathcal{M}=\mathcal{M}_{0}+\mathcal{E}$ where

$$
\begin{aligned}
& \mathcal{M}_{0}=\sum_{k \leq M} \frac{y_{k}}{k} \sum_{m \leq \frac{k T}{2 \pi}} a(m) \frac{\mu(k /(m, k))}{\phi(k /(m, k))}, \\
& \mathcal{E}=\sum_{k \leq M} \frac{y_{k}}{k} \sum_{m \leq \frac{k T}{2 \pi}} a(m) \sum_{\substack{q \mid k \\
q>1}} \sum_{\psi \bmod q}^{*} \tau(\bar{\psi}) \sum_{\substack{d|m \\
d| k}} \psi\left(\frac{m}{d}\right) \delta(q, k, d, \psi)
\end{aligned}
$$

Thus we conclude by (15) and above decomposition of $\mathcal{M}$ that

$$
S=S_{R}-\overline{\mathcal{M}_{0}}-\overline{\mathcal{E}}+O\left(T^{1 / 2} \mathscr{L}^{4}\|\mathbf{y}\|_{1}\|\mathbf{x}\|_{\infty}\right) .
$$


The remainder of the article will be devoted to computing asymptotic expressions for $S_{R}$ and $\mathcal{M}_{0}$ and for providing an upper bound for $\mathcal{E}$. The evaluation of $S_{R}$ is straightforward and will be done in the next section. The evaluation of $\mathcal{M}_{0}$ is also essentially elementary. The most involved part of the argument will be in bounding $\mathcal{E}$. In fact, we shall establish the following results which will imply our theorem:

Proposition 4.2. We have

$$
S_{R}=\frac{T}{2 \pi} \sum_{n u \leq M} \frac{r_{0}(n) x_{u} y_{n u}}{n u}+O\left(T^{\epsilon}\left(\|\mathbf{y}\|_{\infty} M+\|\mathbf{y}\|_{1}\right)\right)
$$

where $r_{0}(n)=P_{2}\left(\log \left(\frac{T}{2 \pi}\right)\right)-2 P_{1}\left(\log \left(\frac{T}{2 \pi}\right)\right)(\log n)+(\Lambda * \log )(n)$ and $P_{2}, P_{1}$ are monic polynomials of degrees 2,1 respectively.

Proposition 4.3. (i) We have in the divisor case

$$
\mathcal{M}_{0}=\frac{T}{2 \pi} \sum_{\substack{u, v \leq M \\(u, v)=1}} \frac{c(u, v) H(M ; u, v)}{u v}+\frac{T}{4 \pi} \sum_{g v \leq M} \frac{y_{g} x_{g v}}{g v} R_{2}\left(\log \left(\frac{T}{2 \pi v}\right)\right)+\mathcal{E}_{0}
$$

where

$$
\mathcal{E}_{0} \ll T \exp \left(-c_{4} \sqrt{\log T}\right)\left\|\frac{x_{n}}{n}\right\|_{1}\left\|\frac{\left(\tau_{3} *|x|\right)(n) y_{n}}{n}\right\|_{1},
$$

$H(M ; u, v)=\sum_{g \leq \min (M / u, M / v)} \frac{y_{u g} x_{v g}}{g}$,

$$
c(u, v)=-\frac{1}{2} \Lambda_{2}(a)+R_{1}\left(\log \left(\frac{T}{2 \pi v}\right)\right) \Lambda(u)+\tilde{R}_{1}\left(\log \left(\frac{T}{2 \pi v}\right)\right) \alpha_{1}(u)+\alpha_{2}(u),
$$

and $R_{1}, \tilde{R}_{1}, R_{2}$ are monic polynomials of degrees $1,1,2$. Moreover, $\alpha_{1}, \alpha_{2}$ are arithmetic functions supported on those $n$ with $\omega(n) \leq 2$. More precisely, $\alpha_{1}\left(p^{\alpha}\right)=\frac{\log p}{p-1}, \alpha_{2}\left(p^{\alpha}\right)=-\frac{(\alpha+1)(\log p)^{2}}{p-1}+\frac{D \log p}{p-1}-\frac{\log p}{(p-1)^{2}}$ for some $D \in \mathbb{R}$, and $\alpha_{2}\left(p^{\alpha} q^{\beta}\right)=-(\log p)(\log q)\left(\frac{1}{p-1}+\frac{1}{q-1}\right)$ for $\alpha, \beta \in \mathbb{N}$.

(ii) In the resonator case, we have, assuming the large zero-free region conjecture for the Riemann zeta function, the same result as above but with

$$
\mathcal{E}_{0} \ll T \exp \left(-\frac{c_{4}^{\prime} \log T}{\log \log T}\right)\left\|\frac{x_{n}}{n}\right\|_{1}\left\|\frac{\left(\tau_{3} *|x|\right)(n) y_{n}}{n}\right\|_{1} .
$$

Theorem 4.4. (i) If $x_{n}, y_{n}$ satisfy (2), (3) then for $0<\theta<1 / 2$ we have for any $A^{\prime}>0$

$$
\mathcal{E} \ll_{A^{\prime}} T(\log T)^{-A^{\prime}}+T^{\frac{3}{4}+\frac{\theta}{2}+\epsilon} .
$$

(ii) Assume the large zero-free region conjecture. If $x_{n}=y_{n}=f(n)$ where $f$ is defined by (4) then for $0<\theta<1 / 6$ we have

$$
\mathcal{E} \ll T \exp \left(-\frac{c_{5} \log T}{\log \log T}\right)+T^{\frac{3}{4}+\frac{3 \theta}{2}+\epsilon} .
$$


By (21), Propositions 4.2,4.4, and the bounds for the coefficients given in section 3 we obtain

$$
\begin{aligned}
S & =\frac{T}{2 \pi} \sum_{n u \leq M} \frac{x_{u} x_{n u} r_{0}(n)}{n u}-\frac{T}{4 \pi} \sum_{g v \leq M} \frac{y_{g} x_{g v}}{g v} R_{2}\left(\log \left(\frac{T}{2 \pi v}\right)\right) \\
& -\frac{T}{2 \pi} \sum_{\substack{u, v \leq M \\
(u, v)=1}} \frac{c^{\prime}(u, v) H(M ; u, v)}{u v}+\mathcal{E}_{0}+\mathcal{E}+O\left(T^{\epsilon}\left(\|\mathbf{y}\|_{\infty} M+\|\mathbf{y}\|_{1}\right)\right)
\end{aligned}
$$

where $\mathcal{E}_{0}$ and $\mathcal{E}$ are as in the proceeding propositions. Setting $r_{1}(u, v)=$ $-c^{\prime}(u, v)$ we see that we obtain the principal term of Theorem 1.3. By (23) and (6) we obtain an error term of the form $T(\log T)^{-A^{\prime}}+T^{\frac{3}{4}+\frac{\theta}{2}+\epsilon}$ as asserted. By (24) and (9) we obtain the error term $T \exp \left(-\frac{c_{1} \log T}{\log \log T}\right)+T^{\frac{3}{4}+\frac{\theta}{2}+\epsilon}$.

\section{Evaluation of $S_{R}$ : Proof of Proposition 4.2}

In this section we evaluate the term $S_{R}$. Recall that

$S_{R}=\frac{1}{2 \pi i} \int_{\kappa+i}^{\kappa+i T}\left(\frac{\chi^{\prime}}{\chi}(s)^{2} \zeta(s)-2 \frac{\chi^{\prime}}{\chi}(s) \zeta^{\prime}(s)+\frac{\zeta^{\prime}}{\zeta}(s) \zeta^{\prime}(s)\right) X(s) Y(1-s) d s$.

The above integral will be evaluated by considering the more general expression:

$$
J_{k}=J_{k}(T)=\frac{1}{2 \pi i} \int_{\kappa+i}^{\kappa+i T}\left(\frac{\chi^{\prime}}{\chi}(s)\right)^{k} D(s) X(s) Y(1-s) d s
$$

where $D(s)=\sum_{n=1}^{\infty} \alpha_{n} n^{-s}$ and $k \in \mathbb{Z}_{\geq 0}$. Suppose that $\sum_{n=1}^{\infty}\left|\alpha_{n}\right| n^{-\sigma} \ll$ $(\sigma-1)^{-\alpha}$ as $\sigma \rightarrow 1$. We will establish:

Lemma 5.1. Suppose $\left|\alpha_{n}\right| \ll n^{\epsilon}$ and we have coefficients $x_{n}, y_{n}$ satisfying $\left\|x_{n} / n\right\|_{1},\left\|y_{n} / n\right\|_{1} \ll T^{\epsilon}$. Then for $k \in \mathbb{N}$

$$
J_{k}=\frac{(-1)^{k} T P_{k}\left(\log \left(\frac{T}{2 \pi}\right)\right)}{2 \pi} \sum_{n u \leq M} \frac{\alpha_{n} x_{u} y_{n u}}{n u}+O\left(T^{\epsilon}\left(\|\mathbf{y}\|_{\infty} M+\|\mathbf{y}\|_{1}\right)\right)
$$

where $P_{k}$ is a monic polynomial of degree $k$.

Proof of Proposition 4.2. By our expression for $S_{R}$ above it suffices to apply Lemma 5.1 in the cases $k=2, \alpha_{n}=1, k=1, \alpha_{n}=-(\log n)$, and $k=0, \alpha_{n}=$ $(\Lambda * \log )(n)$. Thus

$$
S_{R} \sim \frac{T}{2 \pi} \sum_{n u \leq M} \frac{x_{u} y_{n u}}{n u}\left(P_{2}\left(\log \left(\frac{T}{2 \pi}\right)\right)-2 P_{1}\left(\log \left(\frac{T}{2 \pi}\right)\right)(\log n)+(\Lambda * \log )(n)\right)
$$

with an error $O\left(T^{\epsilon}\left(\|\mathbf{y}\|_{\infty} M+\|\mathbf{y}\|_{1}\right)\right.$ as claimed.

Proof of Lemma 5.1. We have the estimate $\frac{\chi^{\prime}}{\chi}(s)=-\log \frac{|t|}{2 \pi}+O(1 /(1+|t|))$ valid for $1 / 2 \leq \sigma \leq 2$ and $t \geq 1$. Thus

$$
\left.J_{k}=\frac{1}{2 \pi i} \int_{\kappa+i}^{\kappa+i T}(-\log (t / 2 \pi))^{k}+O_{k}\left(\mathscr{L}^{k-1} t^{-1}\right)\right) D(s) X(s) Y(1-s) d s .
$$


One checks that the error term contributes $\ll T^{\epsilon}\|\mathbf{y}\|_{1}$. Exchanging summation and integration order yields

$$
J_{k}=\sum_{n, u, v} \frac{\alpha_{n} x_{u} y_{v}(-1)^{k}}{n^{\kappa} u^{\kappa} v^{1-\kappa} 2 \pi} \int_{1}^{T}(\log (t / 2 \pi))^{k}\left(\frac{v}{n u}\right)^{i t} d t+O\left(T^{\epsilon}\|\mathbf{y}\|_{1}\right)
$$

where $s=\kappa+i t$. We now write $J_{k}=J_{d}+J_{n d}$ where $J_{d}$ consists of the the diagonal terms $v=n u$ and $J_{n d}$ consists of the terms $v \neq n u$. We have

$$
J_{d}=\frac{(-1)^{k}}{2 \pi} \sum_{n u \leq M} \frac{\alpha_{n} x_{u} y_{n u}}{n u} \int_{1}^{T} \log ^{k}(t / 2 \pi) d t .
$$

It is simple to see that $\int_{1}^{T} \log ^{k}\left(\frac{t}{2 \pi}\right) d t=T P_{k}\left(\log \left(\frac{T}{2 \pi}\right)\right)+O_{k}(1)$ where $P_{k}$ is monic of degree $k$. Moreover, since $\left|\alpha_{n}\right|,\left\|\frac{x_{n}}{n}\right\|_{1},\left\|\frac{y_{n}}{n}\right\|_{1} \ll T^{\epsilon}$ we have

$$
J_{d}=\frac{(-1)^{k} T P_{k}\left(\log \left(\frac{T}{2 \pi}\right)\right)}{2 \pi} \sum_{n u \leq M} \frac{\alpha_{n} x_{u} y_{n u}}{n u}+O\left(T^{\epsilon}\right) .
$$

The remainder term is

$$
J_{n d}=\sum_{\substack{n, u, v \\ v \neq n u}} \frac{\alpha_{n} x_{u} y_{v}}{n^{\kappa} u^{\kappa} v^{1-\kappa}} \frac{1}{2 \pi} \int_{1}^{T} \log ^{k}(t / 2 \pi)\left(\frac{v}{n u}\right)^{i t} d t .
$$

For $v \neq n u$ the integral is $\ll \mathscr{L}^{k}\left(\left|\log \frac{v}{n u}\right|\right)^{-1}$ and hence

$$
J_{n d} \ll \mathscr{L}^{k+\alpha}|| x_{n} / n \|_{1} \sum_{\substack{v \leq M \\ v \neq n u}} \frac{\left|y_{v}\right|}{v^{1-\kappa}\left|\log \left(\frac{v}{n u}\right)\right|} .
$$

Since $\kappa=1+O\left(\mathscr{L}^{-1}\right)$ it suffices to bound $S(h)=\sum_{\substack{v \leq M \\ v \neq h}}\left|y_{v}\right|\left|\log \frac{v}{h}\right|^{-1}$. If $h \geq 1.1 M$, we have $S(h) \ll\|\mathbf{y}\|_{1}$. We now suppose $h<1.1 M$. The contribution to $S(h)$ from those $v \geq 1.5 h$ and $v \leq 0.5 h$ is bounded by $\|\mathbf{y}\|_{1}$. Consider the interval $I=[0.5 j, 1.5 j] \cap[1, M]$. For those integers $k$ not in this interval we obtain

$$
\sum_{k \notin I} \frac{\left|y_{k}\right|}{|\log (k / j)|} \ll \sum_{k \leq j / 2}\left|y_{k}\right|+\sum_{3 j / 2 \leq k \leq M}\left|y_{k}\right| \ll\|\mathbf{y}\|_{1} .
$$

Either $I=[j / 2,3 j / 2],[1,3 j / 2],[j / 2, M]$. In the first case

$$
\begin{aligned}
& \sum_{k \in I} \frac{\left|y_{k}\right|}{|\log (j / k)|}=\sum_{s=1}^{j / 2} \frac{\left|y_{j-s}\right|}{|\log (j /(j-s))|}+\sum_{s=1}^{j / 2} \frac{\left|y_{j+s}\right|}{|\log (j /(j+s))|} \\
& \ll j \sum_{s=1}^{j / 2} \frac{\left|y_{j-s}\right|}{s}+\left.j \sum_{s=1}^{j / 2} \frac{\left|y_{j+s}\right|}{s} \ll|| \mathbf{y}\right|_{\infty} j(\log j) .
\end{aligned}
$$

The argument for the second and third cases is analogous. We deduce $J_{n d} \ll T^{\epsilon}\left(\|\mathbf{y}\|_{\infty} M+\|\mathbf{y}\|_{1}\right)$ and thus the lemma is established. 


\section{Preliminary Lemmas}

In the section we prove several lemmas that will aid us in evaluating $\mathcal{M}_{0}$ and bounding $\mathcal{E}$. Lemmas 6.1 and 6.5 will be applied in our evaluation of $\mathcal{M}_{0}$. Lemmas 6.1, 6.4, 6.6, and 6.7 shall be invoked when we bound $\mathcal{E}$. The next lemma tells us how to decompose an arithmetic function which is the convolution of other arithmetic functions.

Lemma 6.1. Let $f_{1}, f_{2}, \ldots, f_{j}$ be arithmetic functions and let $D \in \mathbb{N}$. Given a decomposition $D=d_{1} d_{2} \cdots d_{j}$ we define the integers $D_{i}=\prod_{u=1}^{j-i} d_{u}$ for $1 \leq i \leq j-1$ and $D_{j}=1$. We have the identities

$$
\begin{aligned}
& \sum_{\substack{m \leq X \\
(m, k)=1}}\left(f_{1} * f_{2} * \cdots * f_{j}\right)(m D) \\
&= \sum_{\substack{d_{1} d_{2} \cdots d_{j}=D \\
m_{1}\left(m_{2} \cdots m_{j} \leq X \\
\left(m_{i}, k D_{i}\right)=1\right.}} f_{1}\left(m_{1} d_{j}\right) f_{2}\left(m_{2} d_{j-1}\right) \cdots f_{j}\left(m_{j} d_{1}\right) \\
& \sum_{(m, k)=1} \frac{\left(f_{1} * f_{2} * \cdots * f_{j}\right)(m D)}{m^{s}}=\sum_{d_{1} d_{2} \cdots d_{j}=D} \prod_{i=1}^{j} \sum_{\left(m_{i}, k D_{i}\right)=1} \frac{f_{i}\left(m_{i} d_{j-i}\right)}{m_{i}^{s}} .
\end{aligned}
$$

Proof. The proof of this argument follows the proof of Lemma 3 of [3] (p. 506). The case $j=2$ follows from the identity

$$
\left(f_{1} * f_{2}\right)(m D)=\sum_{g \mid D} \sum_{\substack{h \mid m \\\left(h, \frac{D}{g}\right)=1}} f_{1}(g h) f_{2}\left(\frac{m D}{g h}\right) .
$$

By making the identifications $g=d_{2}, \frac{D}{g}=d_{1}, h=m_{1}$, and $\frac{m}{h}=m_{2}$ we obtain (27), (28) for $j=2$. For $j>2$ the assertion follows by induction.

We now introduce some arithmetic functions that will appear in our evaluation of $\mathcal{M}_{0}$. We define $\eta_{1}(k)=\sum_{p \mid k} \frac{\log p}{p-1}, \eta_{2}(k)=-\sum_{p \mid k} \frac{p \log p}{(p-1)^{2}}$,

$$
g(h, k)=\sum_{\substack{a \mid h, a=p^{t} \\(a, k)=1}} \frac{\Lambda(a) \log p}{p-1} .
$$

Moreover, we define arithmetic functions $\phi_{j}(n)$ for $j=1 \ldots 4$ as follows:

$$
\begin{aligned}
\phi_{1}(n)=\sum_{k \mid n} \mu(k) \eta_{1}(k), \phi_{2}(n) & =\sum_{k \mid n} \mu(k) \eta_{2}(k), \\
\phi_{3}(n)=\sum_{k \mid n} \mu(k) g(h, k), \phi_{4}(n) & =\sum_{k \mid n} \mu(k) \eta_{1}(k) \log k .
\end{aligned}
$$

We prove that the $\phi_{j}$ are supported on integers $n$ such that $\omega(n) \leq 2$. 
Lemma 6.2. $\phi_{1}, \phi_{2}, \phi_{3}$ are supported only on the prime powers and are given by:

$$
\phi_{1}\left(p^{\alpha}\right)=-\frac{\log p}{p-1}, \phi_{2}\left(p^{\alpha}\right)=\frac{p \log p}{(p-1)^{2}}, \phi_{3}\left(p^{\alpha}\right)=\frac{\log p}{p-1} \log \left(p^{\alpha}\right)
$$

for $\alpha \geq 1$. However, $\phi_{4}$ is supported on those $n$ with $\omega(n) \leq 2$ and is given by

$$
\phi_{4}\left(p^{\alpha} q^{\beta}\right)=(\log p)(\log q)\left(\frac{1}{p-1}+\frac{1}{q-1}\right), \phi_{4}\left(p^{\alpha}\right)=-\frac{(\log p)^{2}}{p-1}
$$

where $\alpha, \beta \geq 1$.

Proof. These formulae for the $\phi_{i}$ may be proved directly from their definitions, however it is simpler to employ generating functions. Put $A(s)=$ $\sum_{n=1}^{\infty} \phi_{4}(n) n^{-s}$ where $\phi_{4}(n)=\sum_{h k=n} \mu(k)\left(\sum_{p \mid k} f(p)\right) \log k$ and $f(x)=$ $\frac{\log x}{x-1}$. We have

$$
\begin{aligned}
A(s) & =\sum_{h, k \geq 1} \frac{\mu(k) \log k}{h^{s} k^{s}} \sum_{p \mid k} f(p)=\sum_{p} f(p) \sum_{h=1}^{\infty} \frac{1}{h^{s}} \sum_{p \mid k} \frac{\mu(k) \log k}{k^{s}} \\
& =\sum_{p} \frac{f(p) \mu(p)}{p^{s}} \zeta(s) \sum_{\left(k_{1}, p\right)=1} \frac{\mu\left(k_{1}\right) \log \left(p k_{1}\right)}{k_{1}^{s}} \\
& =\sum_{p} \frac{f(p) \mu(p)}{p^{s}} \zeta(s)\left(\frac{\log p}{1-\frac{1}{p^{s}}} \zeta(s)^{-1}+\sum_{\left(k_{1}, p\right)=1} \frac{\mu\left(k_{1}\right) \log \left(k_{1}\right)}{k_{1}^{s}}\right) .
\end{aligned}
$$

It may be verified that

$$
\sum_{\left(k_{1}, p\right)=1} \frac{\mu\left(k_{1}\right) \log \left(k_{1}\right)}{k_{1}^{s}}=\left(1-1 / p^{s}\right)^{-2} \frac{\log p}{p^{s}} \zeta(s)^{-1}+\left(1-1 / p^{s}\right)^{-1} \frac{\zeta^{\prime}(s)}{\zeta(s)^{2}}
$$

and thus

$$
\begin{aligned}
& A(s)=\sum_{p} \frac{f(p) \mu(p)}{p^{s}}\left(\frac{\log p}{\left(1-1 / p^{s}\right)}+\frac{\log p}{p^{s}\left(1-1 / p^{s}\right)^{2}}+\frac{1}{\left(1-1 / p^{s}\right)} \frac{\zeta^{\prime}(s)}{\zeta(s)}\right) \\
& =\sum_{p^{j} ; j \geq 1} \frac{f(p) \mu(p) \log p}{p^{j s}}+\sum_{p^{j} ; j \geq 2} \frac{f(p) \mu(p) \log p(j-1)}{p^{j s}}+\sum_{p^{j}} \frac{f(p) \mu(p)}{p^{j s}} \frac{\zeta^{\prime}(s)}{\zeta(s)} \\
& =\sum_{p^{j}} \frac{f(p) \mu(p) \log \left(p^{j}\right)}{p^{j s}}+\left(\sum_{p^{j}} \frac{f(p)}{p^{j s}}\right)\left(-\frac{\zeta^{\prime}(s)}{\zeta(s)}\right) .
\end{aligned}
$$

Let $\theta(n)$ be supported on prime powers defined by $\theta\left(p^{j}\right)=f(p)$. We write

$$
\left(\sum_{p^{j}} \frac{f(p)}{p^{j s}}\right)\left(-\frac{\zeta^{\prime}(s)}{\zeta(s)}\right)=\sum_{n \geq 1} \frac{t(n)}{n^{s}}
$$


with $t(n)=(\theta * \Lambda)(n)$. It follows easily that $\phi_{4}$ is supported on those $n$ such that $\omega(n) \leq 2$. We begin by supposing that $\omega(n)=2$ and $n=p^{\alpha} q^{\beta}$. Thus

$$
t\left(p^{\alpha} q^{\beta}\right)=\sum_{u v=p^{\alpha} q^{\beta}} \Lambda(u) \theta(v)=(\log p) f(q)+(\log q) f(p) .
$$

Next for $n=p^{\alpha}$

$$
t\left(p^{\alpha}\right)=\sum_{u v=p^{\alpha}} \Lambda(u) \theta(v)=\sum_{i+j=\alpha, i, j \geq 1} \log p f(p)=(\alpha-1) f(p)(\log p) .
$$

Since $\phi_{4}(n)=f(n) \mu(n) \log (n)+t(n)$ the result follows from (31) and (32).

The next result provides an estimate for divisor sums in short intervals. This is Theorem 2 of [16].

Lemma 6.3. Let $\alpha, \beta$ be real numbers and let $a, q, k$ be integers. Suppose that $0<\alpha, \beta<1 / 2, k \geq 2$, and $(a, k)=1$. We have as $t \rightarrow \infty$,

$$
\sum_{\substack{t-u \leq n \leq t \\ n \equiv a(q)}} \tau_{k}(n) \ll \frac{u}{q}\left(\frac{\phi(q)}{q} \log t\right)^{k}
$$

uniformly in $a, q$, and $t$ provided that $q<t^{1-\alpha}$ and $t^{\beta}<u \leq t$.

We will also require a short interval estimate for $\tau_{k} * x$ where $x$ is an arbitrary arithmetic function.

Lemma 6.4. Let $T \ll w \ll T^{2}$ and $M \leq \sqrt{T}$. Let $x$ be an arithmetic function supported on $[1, M]$. Then

$$
\sum_{t-u \leq n \leq t}\left(\tau_{k} * x\right)(n) \ll u(\log t)^{k-1}\left\|x_{n} / n\right\|_{1}
$$

where $u=w U^{-1}, \exp (c \sqrt{\log w}) \leq U \leq \exp \left(\frac{2 \log w}{\log \log w}\right)$ and $\frac{w}{2} \leq t-u \leq t \leq w$. Proof. Notice that our sum may be rewritten as

$$
\sum_{t-u<n \leq t}\left(\tau_{k} * x\right)(n)=\sum_{b \leq M} x_{b} \sum_{\frac{t}{b}-\frac{u}{b} \leq a \leq \frac{u}{b}} \tau_{k}(b) .
$$

By our conditions on $u$ and $t, \frac{u}{b} \gg\left(\frac{t}{b}\right)^{\epsilon}$ for all $1 \leq b \leq M$. Hence by Lemma 6.3

$$
\sum_{b \leq M} x_{b} \sum_{\frac{t}{b}-\frac{u}{b} \leq a \leq \frac{t}{b}} \tau_{k}(a) \ll u(\log t)^{k-1} \sum_{b \leq M} \frac{x_{b}}{b} \ll u \mathscr{L}^{k-1}\left\|\frac{x_{n}}{n}\right\|_{1} .
$$

The next lemma evaluates asymptotically a sum that will appear in our evaluation of $\mathcal{M}_{0}$. The proof of this lemma is very similar to the asymptotic evaluation of $\sum_{n \leq x} \Lambda(n)$ that occurs in the proof of the prime number theorem. 
Lemma 6.5. Let $h, k \in \mathbb{N}, h, k \leq M$ and $x \geq 1$ such that $\log x \asymp \log T$.

$$
\begin{aligned}
\sum_{\substack{u \leq x \\
(u, k)=1}}(\Lambda * \log )(h u) & =\frac{x \phi(k)}{k}\left(\frac{1}{2}(\log x)^{2}+2 \log (x / e) \log h+(\Lambda * \log )(h)\right. \\
& \left.+\left(C_{0}-\eta_{1}(k)\right) \log (x / e)+C_{1} \eta_{1}(k)-\eta_{2}(k)-g(h, k)\right) \\
& +O\left(\tau(h) x \exp \left(-C_{2} \sqrt{\log T}\right)\right)
\end{aligned}
$$

for some explicit constants $C_{j}$ for $j=0,1,2$ and $\eta_{1}, \eta_{2}, g_{1}, g_{2}$, and $g$ are the arithmetic functions defined above. Note if we further assume that $\zeta(s)$ is non-vanishing in the region $\operatorname{Re}(s) \geq 1-\frac{c_{0}}{\log \log (|\operatorname{Im}(s)|+4)}$ then the above error term may be reduced to

$$
O\left(\tau(h) x \exp \left(-\frac{C_{2}^{\prime} \log T}{\log \log T}\right)\right) .
$$

Proof. Put $A(z)=\sum_{(u, k)=1}(\log * \Lambda)(h u) u^{-z}$. We have by Perron's formula

$$
\sum_{\substack{u \leq x \\(u, k)=1}}(\Lambda * \log )(h u)=\frac{1}{2 \pi i} \int_{\kappa-i U}^{\kappa+i U} A(z) x^{z} \frac{d z}{z}+O\left(x^{\kappa} \sum_{n=1}^{\infty} \frac{(\Lambda * \log )(h n)}{n^{\kappa}(1+T|\log (x / n)|)}\right)
$$

for $\kappa=1+O\left((\log x)^{-1}\right)$ (see [18, p. 132]). First note that $(\Lambda * \log )(h n) \ll$ $\tau(h)^{2}(\log n)^{2} \tau(n)^{2}$. In the last sum above the contribution from those $n$ not in $[0.5 x, 2 x]$ is

$$
\ll \frac{\tau(h) x}{U} \sum_{n \geq 1} \frac{(\log n)^{2} \tau(n)^{2}}{n^{\kappa}} \ll \frac{\tau(h) x(\log x)^{6}}{U} .
$$

We now consider the contribution from those $n \in[0.5 x, 2 x]$. We begin with the interval $[0.5 x, x)$. This yields the contribution

$$
\ll \tau(h) \mathscr{L}^{2} \sum_{\frac{x}{2} \leq n<x} \tau(n) \min \left(1, U^{-1}|\log (x / n)|^{-1}\right) .
$$

Since $|\log (x / n)|^{-1} \ll \frac{x}{|x-n|}$ this sum is

$$
\ll \sum_{x-\frac{x}{U} \leq n<x} \tau(n)+\frac{x}{U} \sum_{\frac{x}{2}<n<x-\frac{x}{U}} \frac{\tau(n)}{x-n} .
$$

By an application of Lemma 6.3 the first sum is $\ll \frac{x}{U} \log x$. Now dividing the second sum into $K \ll U$ intervals of length $\frac{x}{U}$ and invoking again Lemma 6.3 we see that

$$
\begin{aligned}
& \frac{x}{U} \sum_{\frac{x}{2}<n<x-\frac{x}{U}} \frac{\tau(n)}{x-n} \ll \frac{x}{U} \sum_{k=1}^{K} \frac{U}{k x} \sum_{x-(k+1) \frac{x}{U} \leq n<x-k \frac{x}{U}} \tau(n) \\
& \ll \sum_{k=1}^{K} k^{-1} \frac{x \log x}{U} \ll \frac{x \log x \log U}{U} .
\end{aligned}
$$


Combining estimates we deduce for $U \leq x$

$$
\sum_{\substack{u \leq x \\(u, k)=1}}(\Lambda * \log )(h u)=\frac{1}{2 \pi i} \int_{\kappa-i U}^{\kappa+i U} A(z) x^{z} \frac{d z}{z}+O\left(\frac{\tau(h) x \mathscr{L}^{6}}{U}\right) .
$$

In a moment we shall give a decomposition of $A(z)$ in terms of other well known Dirichlet series and thus we shall show that it has a triple pole at $z=1$. Let $\sigma_{0}(t)=1-\frac{c^{\prime}}{\log (|t|+2)}+i t$ for $t \in \mathbb{R}$. We shall shift the contour left to $\operatorname{Re}(s)=\sigma_{0}(U)$. Therefore

$$
\begin{aligned}
& \sum_{\substack{u \leq x \\
(u, k)=1}}(\Lambda * \log )(h u)=\operatorname{Re}_{z=1}\left(A(z) x^{z} z^{-1}\right)+\frac{1}{2 \pi i} \int_{\sigma_{0}(U)-i U}^{\sigma_{0}(U)+i U} A(z) x^{z} \frac{d z}{z} \\
& +O\left(\left(\int_{\kappa+i U}^{\sigma_{0}(U)+i U}+\int_{\kappa-i U}^{\sigma_{0}(U)-i U}\right) A(z) x^{z} \frac{d z}{z}+\frac{\tau(h) x \mathscr{L}^{6}}{U}\right) .
\end{aligned}
$$

By Lemma 6.1

$$
A(z)=\sum_{a b=h} \sum_{(c, a k)=1} \frac{\log (b c)}{c^{z}} \sum_{(d, k)=1} \frac{\Lambda(a d)}{d^{z}}:=\sum_{a b=h} A_{1}(z ; a, b) A_{2}(z ; a) .
$$

A calculation shows that

$$
A_{1}(z ; a, b)=\Phi(z ; a k)\left(\log (b) \zeta(z)-\zeta^{\prime}(z)-\zeta(z) \eta(z ; a k)\right)
$$

where for $n \in \mathbb{N}$

$$
\Phi(z ; n)=\prod_{p \mid n}\left(1-p^{-z}\right), \eta(z ; n):=\frac{\Phi^{\prime}(z ; n)}{\Phi(z ; n)}=\sum_{p \mid n} \frac{\log p}{p^{z}-1} .
$$

Also

$$
A_{2}(z ; a)= \begin{cases}-\frac{\zeta^{\prime}}{\zeta}(z)-\eta(z ; k) & \text { if } a=1 \\ \frac{\log p}{1-p^{-z}} & \text { if } a=p^{l},(a, k)=1 \\ \log p & \text { if } a=p^{l}, p \mid k \\ 0 & \text { else }\end{cases}
$$

It is convenient to define $\tilde{\Lambda}(a ; z)=(\log p) /\left(1-p^{-z}\right)=\Lambda(a)\left(1+\frac{1}{p^{z}-1}\right)$ for $a=p^{l}$. Thus we have $A(z)=B_{1}(z)+B_{2}(z)+B_{3}(z)$ where

$$
B_{1}(z)=\left(-\frac{\zeta^{\prime}}{\zeta}(z)-\eta(z ; k)\right) \Phi(z ; k)\left(-\zeta^{\prime}(z)+\zeta(z)(\log h-\eta(z ; k))\right.
$$

$$
B_{2}(z)=\sum_{a \mid(h, k)} \Lambda(a) \Phi(z ; a k)\left(-\zeta^{\prime}(z)+\zeta(z)(\log (h / a)-\eta(z ; a k))\right)
$$

$$
B_{3}(z)=\sum_{\substack{a \mid h \\(a, k)=1}} \tilde{\Lambda}(a ; z) \Phi(z ; a k)\left(-\zeta^{\prime}(z)+\zeta(z)(\log (h / a)-\eta(z ; a k))\right)
$$


We define $R_{j}=\operatorname{Res}_{z=1}\left(B_{j}(z) x^{z} / z\right)$ for $j=1,2,3$. We remark that $\eta(1 ; k)=$ $\eta_{1}(k), \eta^{\prime}(1 ; k)=\eta_{2}(k), \Phi(1 ; k)=\frac{\phi(k)}{k}$,

$$
\begin{aligned}
& \Phi^{\prime}(1 ; k)=\Phi(1 ; k) \eta_{1}(k)=\frac{\phi(k)}{k} \eta_{1}(k), \\
& \Phi^{(2)}(k)=\Phi(1 ; k)\left(\eta(1 ; k)^{2}+\eta^{\prime}(1 ; k)\right)=\frac{\phi(k)}{k}\left(\eta_{1}(k)^{2}+\eta_{2}(k)\right) .
\end{aligned}
$$

We now list several Laurent series that we require in the residue computation

$$
\begin{aligned}
\frac{x^{z}}{z} & =x\left(1+\log (x / e)(z-1)+\left(\frac{1}{2}(\log x)^{2}-\log (x / e)\right)(z-1)^{2}+\cdots\right), \\
\Phi(z ; n) & =\Phi(1 ; n)+\Phi^{\prime}(1 ; n)(z-1)+\frac{1}{2} \Phi^{(2)}(1 ; n)(z-1)^{2}+\cdots, \\
\frac{\zeta^{\prime}(z)^{2}}{\zeta(z)} & =\frac{1}{(z-1)^{3}}\left(1+a_{1}(z-1)+a_{2}(z-1)^{2}+\cdots\right), \\
-\zeta^{\prime}(z) & =\frac{1}{(z-1)^{2}}\left(1+b_{2}(z-1)^{2}+\cdots\right),
\end{aligned}
$$

with $a_{j}, b_{j} \in \mathbb{R}$. Note that

$$
B_{1}(z)=\Phi(z ; k)\left(\frac{\zeta^{\prime}(z)^{2}}{\zeta(z)}-\zeta^{\prime}(z)(\log (h)-2 \eta(z ; k))+\zeta(z)\left(\eta(z ; k)^{2}-\log (h) \eta(z ; k)\right)\right) .
$$

We begin by writing $R_{1}=R_{11}+R_{12}+R_{13}$ where

$$
\begin{aligned}
& R_{11}=\operatorname{Res}_{z=1}\left(\Phi(z ; k) \frac{\zeta^{\prime}(z)^{2}}{\zeta(z)} \frac{x^{z}}{z}\right), \\
& R_{12}=\operatorname{Res}_{z=1}\left(-\zeta^{\prime}(z) \Phi(z ; k)(\log (h)-2 \eta(z ; k)) \frac{x^{z}}{z}\right), \\
& R_{13}=\operatorname{Res}_{z=1}\left(\zeta(z) \Phi(z ; k)\left(\eta(z ; k)^{2}-\log (h) \eta(z ; k)\right) \frac{x^{z}}{z}\right) .
\end{aligned}
$$

We deduce from the above Laurent series that

$$
\begin{aligned}
R_{11} & =x\left((1 / 2) \log (x)^{2} \Phi(1 ; k)+\log (x / e)\left(\left(a_{1}-1\right) \Phi(1 ; k)+\Phi^{\prime}(1 ; k)\right)\right. \\
& +\left(a_{2} \Phi(1 ; k)+a_{1} \Phi^{\prime}(1 ; k)+\Phi^{(2)}(1 ; k)\right) .
\end{aligned}
$$

By (37) this simplifies to

$$
R_{11}=\frac{x \phi(k)}{k}\left(\frac{1}{2} \log (x)^{2}+\log (x / e)\left(\left(a_{1}-1\right)+\eta_{1}(k)\right)+\left(a_{2}+a_{1} \eta_{1}(k)+\eta_{1}(k)^{2}+\eta_{2}(k)\right)\right) .
$$

Similar calculations yield

$$
R_{12}=\frac{x \phi(k)}{k}\left(\left(\log (x / e)+\eta_{1}(k)\right)\left(\log h-2 \eta_{1}(k)\right)-2 \eta_{2}(k)\right)
$$

and $R_{13}=\frac{x \phi(k)}{k}\left(\eta_{1}(k)^{2}-\eta_{1}(k) \log h\right)$. Combining our formulae we have

$$
R_{1}=\frac{1}{2}(\log x)^{2}+\log (x / e)\left(\log h+a_{1}-1-\eta_{1}(k)\right)+a_{1} \eta_{1}(k)-\eta_{2}(k) .
$$


We next deal with $R_{2}$. Note that

$$
\operatorname{Res}_{z=1}\left(-\zeta^{\prime}(z) \Phi(z ; a k) \frac{x^{z}}{z}\right)=x \Phi(1 ; a k)\left(\log (x / e)+\eta_{1}(a k)\right)
$$

$\operatorname{Res}_{z=1}\left(\zeta(z) \Phi(z ; a k)(\log (h / a)-\eta(z ; a k)) \frac{x^{z}}{z}\right)=x \Phi(1 ; a k)\left(\log (h / a)-\eta_{1}(a k)\right)$, and thus

$$
R_{2}=x \sum_{a \mid(h, k)} \Lambda(a) \frac{\phi(a k)}{a k}(\log (x / e)+\log (h / a)) .
$$

Now observe that $\frac{\phi(a k)}{a k}=\frac{\phi(k)}{k}$ if $a \mid k$ and $\frac{\phi(a k)}{a k}=\frac{\phi(k)}{k}(1-1 / p)$ if $(a, k)=1$ and hence

$$
R_{2}=\frac{x \phi(k)}{k} \sum_{a \mid(h, k)} \Lambda(a)(\log (x / e)+\log (h / a)) .
$$

We now consider $R_{3}$. Since

$$
\begin{aligned}
& \operatorname{Res}_{z=1}\left(-\zeta^{\prime}(z) \tilde{\Lambda}(a, z) \Phi(z ; a k)\right) \\
& =x \Phi(1 ; a k)\left(\tilde{\Lambda}(a ; 1) \log (x / e)+\tilde{\Lambda}^{\prime}(a ; 1)+\tilde{\Lambda}(a ; 1) \eta_{1}(a k)\right), \\
& \operatorname{Res}_{z=1}(\zeta(z) \tilde{\Lambda}(a ; z) \Phi(z ; a k)(\log (h / a)-\eta(z ; a k))) \\
& =x \Phi(1 ; a k) \tilde{\Lambda}(a ; 1)\left(\log (h / a)-\eta_{1}(a k)\right),
\end{aligned}
$$

it follows that

$$
R_{3}=x \sum_{\substack{a \mid h \\(a, k)=1}} \Phi(1 ; a k)\left(\tilde{\Lambda}(a ; 1)(\log (x / e)+\log (h / a))+\tilde{\Lambda}^{\prime}(a ; 1)\right) .
$$

By the identities $\Phi(1 ; a k)=\frac{\phi(a k)}{a k}=\frac{\phi(k)}{k}(1-1 / p), \tilde{\Lambda}(a ; 1)=\Lambda(a) \frac{p}{p-1}$, $\tilde{\Lambda}^{\prime}(a ; 1)=-\Lambda(a) \frac{p \log p}{(p-1)^{2}}$ we derive

$$
R_{3}=\frac{x \phi(k)}{k} \sum_{\substack{a \mid h \\(a, k)=1}} \Lambda(a)\left(\log (x / e)+\log (h / a)-\frac{\log p}{p-1}\right) .
$$

Combining $R_{2}$ and $R_{3}$ we have

$$
\begin{aligned}
R_{2}+R_{3} & =\frac{x \phi(k)}{k}\left(\sum_{a \mid h} \Lambda(a)(\log (x / e)+\log (h / a))-\sum_{\substack{a \mid h \\
(a, k)=1}} \frac{\Lambda(a) \log p}{p-1}\right) \\
& =\frac{x \phi(k)}{k}(\log (x / e) \log h+(\Lambda * \log )(h)-g(h, k)) .
\end{aligned}
$$

Combining our expressions for $R_{1}$ (38) and $R_{2}+R_{3}$ (41) we see that

$$
\begin{aligned}
\text { residue } & =\frac{x \phi(k)}{k}\left(\frac{1}{2}(\log x)^{2}+2 \log (x / e) \log h+(\Lambda * \log )(h)\right. \\
& +\left(C_{0}-\eta_{1}(k)\right) \log (x / e)+a_{1} \eta_{1}(k)-\eta_{2}(k)-g(h, k) .
\end{aligned}
$$


It suffices to compute the other error terms in (33). We have the standard bounds $\left|\left(\zeta^{\prime} / \zeta\right)(z)\right| \ll(\log |z|),\left|\zeta^{(j)}(z)\right| \ll(\log |z|)^{j}$ for $j=1,2, \operatorname{Re}(z) \geq$ $1-\frac{c^{\prime}}{|\operatorname{Im}(z)|}$ and $|\operatorname{Im}(z)| \geq 3$ (see [18, p. 146, p. 158]). Note that by our decomposition $A(z)=B_{1}(z)+B_{2}(z)+B_{3}(z)$ we have

$$
\begin{gathered}
\left|B_{1}(z)\right| \ll\left(\log U+\eta_{1 / 2}(k)\right) j(k)\left(\log ^{2} U+\log U\left(\log h+\eta_{1 / 2}(k)\right),\right. \\
\left|B_{2}(z)+B_{3}(z)\right| \ll j(k) \sum_{a \mid h} \Lambda(a) j(a)\left(\log ^{2} U+\log U\left(\log h+\eta_{1 / 2}(a k)\right)\right)
\end{gathered}
$$

and thus $|A(z)| \ll j(k) \mathscr{L}^{3}$. It follows that the horizontal integrals in (33) are bounded by

$$
\int_{\sigma_{0}(U)}^{c}|A(\sigma \pm i U)| x^{\sigma} \frac{d \sigma}{|\sigma \pm i U|} \ll \frac{x^{c} j(k) \mathscr{L}^{3}}{U} \ll \frac{j(k) x \mathscr{L}^{3}}{U}
$$

and the leftmost vertical integral in (33) is bounded by

$$
x^{\sigma_{0}(U)} \int_{-U}^{U} \frac{\left|A\left(\sigma_{0}(U)+i u\right)\right| d u}{\left|\sigma_{0}(U)+i u\right|} \ll x j(k) \mathscr{L}^{3} \log (U) \exp \left(-\frac{c \log x}{\log (|U|+2)}\right) .
$$

If we choose $U=\exp (\beta \sqrt{\log x})$ for an appropriate $\beta>0$ then these last two error terms are $O\left(j(k) x \exp \left(-\beta^{\prime} \sqrt{\log x}\right)\right)$ for some $\beta^{\prime}>0$. We finally deduce from (33) that

$$
\sum_{\substack{u \leq x \\(u, k)=1}}(\Lambda * \log )(h u)=\text { residue }+O\left((\tau(h)+j(k)) x \exp \left(-C_{2} \sqrt{\log x}\right)\right) .
$$

However, note that one can show $j(k) \ll \exp (o(\sqrt{\log k}))$ and hence the error term can be written as $O\left(\tau(h) \exp \left(-C_{2} \sqrt{\log x}\right)\right)$ for a smaller $C_{2}$.

We give a brief sketch how to adapt this argument for the resonator case assuming the large zero-free region conjecture for $\zeta(s)$. Obviously, the residue term will remain unchanged. Instead in this case, we will move the contour further left to the line $\operatorname{Re}(s)=\sigma_{1}(U)$ where $\sigma_{1}(t)=1-\frac{0.25 c_{0}}{\log \log (|t|+4)}$. In this region, one can establish that $\left|\left(\zeta^{\prime} / \zeta\right)(z)\right| \ll(\log \log |z|),\left|\zeta^{(j)}(z)\right| \ll$ $(\log \log |z|)^{j}$ for $|z| \gg 1$. These results may be proven exactly as in Lemma 6.8 that follows. We deduce

$$
x^{\sigma_{1}(U)} \int_{-U}^{U} \frac{\left|A\left(\sigma_{1}(U)+i u\right)\right| d u}{\left|\sigma_{1}(U)+i u\right|} \ll x j(k) \mathscr{L}^{3} \log (U) \exp \left(-\frac{c \log x}{\log \log (|U|+2)}\right)
$$

on the left edge of the contour. Choosing $U=\exp \left(\frac{\beta \log x}{\log \log x}\right)$ for some $\beta>0$ yields the smaller error term.

We shall require a bound for $\delta$ (18) that occurs in the decomposition (20).

Lemma 6.6. For $d, k, q \in \mathbb{N}, \psi$ a primitive character modulo $q$ and $k q \ll T$ we have

$$
|\delta(q, k q, d, \psi)| \ll \frac{(d, k) \log \log T}{\phi(k) \phi(q)} .
$$

Moreover, if $k q$ is squarefree then this bound may be replaced by $(d, k) /(\phi(k) \phi(q))$. 
Proof. Now for any $a, b \in \mathbb{N}$ we have $\phi(a b) \theta((a, b))=\phi(a) \phi(b)$ where $\theta(n)=$ $\prod_{p \mid n}(1-1 / p)$. However, one can show that $\theta(n) \gg(\log \log |3 n|)^{-1}$. From these observations it follows that

$$
|\delta(q, k q, d, \psi)| \ll \log \log (k q) \sum_{e \mid(d, k)} \frac{\phi(e)}{\phi(k q)} \ll \frac{(d, k) \log \log T}{\phi(q) \phi(k)} .
$$

The second stated bound is obtained by the same method.

Lemma 6.7. Let $h$ be a positive multiplicative function. Let $1 \leq k, q \leq M$. We shall provide a bound for

$$
\theta(\sigma)=\sum_{d \mid k q} \frac{(d, k) h(d)}{d^{\sigma}} .
$$

(i) We first establish:

$$
\theta(\sigma) \ll\left\{\begin{array}{ll}
(1 * h)(k)\left\|\frac{h(n)}{n}\right\|_{1} & \text { if } \sigma=1 \\
\sqrt{k}(1 * h)(k)\|h\|_{\infty} T^{\epsilon} & \text { if } \sigma=1 / 2
\end{array} .\right.
$$

(ii) We assume that $k q$ is squarefree, $h(p) \ll f(p)$ where $f$ is defined by (4), and $q \geq \eta:=\left(\frac{2.5 \mathscr{L}}{\log \mathscr{L}}\right)$. Then we obtain

$$
\theta(\sigma) \ll\left\{\begin{array}{ll}
(1 * h)(k) \exp (o(\sqrt{\mathscr{L}})) & \text { if } \sigma=1 \\
\sqrt{k}(1 * h)(k) T^{\epsilon} & \text { if } \sigma=1 / 2
\end{array} .\right.
$$

Proof. (i) We put $g=(d, k), d=g d_{1}$, and $k=d k_{1}$ so that

$$
\begin{aligned}
& \theta(\sigma)=\sum_{g \mid k} g \sum_{d \mid k q, g=(d, k)} \frac{h(d)}{d^{\sigma}} \ll \sum_{g \mid k} g^{1-\sigma} \sum_{d_{1} \mid q} \frac{h\left(g d_{1}\right)}{d_{1}^{\sigma}} \\
& \ll k^{1-\sigma} \sum_{g \mid k} h(g) \sum_{d_{1} \mid q} \frac{h\left(d_{1}\right)}{d_{1}^{\sigma}} .
\end{aligned}
$$

If $\sigma=1$ then we have the bound $(1 * h)(k)\left\|\frac{h(n)}{n}\right\|_{1}$ and if $\sigma=\frac{1}{2}$ then we apply $\sum_{d_{1} \mid q} \frac{h\left(d_{1}\right)}{\sqrt{d_{1}}} \ll\|h\|_{\infty} T^{\epsilon}$. These bounds prove part (i). For part (ii) $k q$ is squarefree and thus $(k, q)=1$. It follows that

$$
\theta(\sigma)=\sum_{d \mid k} h(d) d^{1-\sigma} \sum_{e \mid q} h(e) e^{-\sigma} \leq k^{1-\sigma}(1 * h)(k) \sum_{e \mid q} h(e) e^{-\sigma} .
$$

Since $q$ is squarefree and $h$ is multiplicative

$$
\log \sum_{e \mid q} \frac{h(e)}{e^{\sigma}}=\sum_{p \mid q} \log \left(1+\frac{h(p)}{p^{\sigma}}\right) \ll \sum_{p \mid q} \frac{h(p)}{p^{\sigma}} \ll \sum_{p \mid q} \frac{f(p)}{p^{\sigma}} .
$$

Noting that $f$ is supported on those $n$ such that $n \geq L^{2}$ we obtain

$$
\sum_{p \mid q, p>L^{2}} \frac{f(p)}{p}=\sum_{p \mid q, p>L^{2}} \frac{L}{p(\log p)} \ll \frac{L \omega(q)}{L^{2} \log L} \ll \frac{\log q}{L \log (L) \log \log q}=o(\sqrt{\log M})
$$


for $L=\sqrt{\log M \log \log M}$ and $q \geq \exp \left(\frac{2.5 \mathscr{L}}{\log \mathscr{L}}\right)$. Now denote the prime divisors of $q$ as $r_{1}, \cdots, r_{k}$. Let $p_{1}, \cdots, p_{k}$ denote the first $k$ primes. We have that

$$
\sum_{p \mid q} \frac{f(p)}{p^{1 / 2}} \leq \sum_{p_{i} \leq 2 \log q} \frac{L}{(\log p) p^{1 / 2}} \ll \frac{L(\log q)^{1 / 2}}{(\log \log q)^{2}}=o(\log T)
$$

for $\exp \left(\frac{2.5 \mathscr{L}}{\log \mathscr{L}}\right) \leq q \leq M$.

The final lemma in this section provides bounds for $L^{(k)}(s, \chi) k=1,2$ and $\frac{L^{\prime}}{L}(s, \chi)$ for $s$ just to the left of $\operatorname{Re}(s)=1$ in the critical strip. We have

Lemma 6.8. Suppose that $\chi$ is a primitive Dirichlet character modulo $q$. For $s=\sigma+i t$ we put $\tau=|t|+4$.

(i) There exists a constant $c>0$ such that if $\operatorname{Re}(s) \geq 1-\frac{c}{\log (q \tau)}$ then

$$
\left|L^{(k)}(s, \chi)\right| \ll \log (q \tau)^{k},\left|\frac{L^{\prime}}{L}(s, \chi)\right| \ll \log (q \tau) .
$$

(ii) Assume the large zero-free region conjecture. If $\operatorname{Re}(s) \geq 1-\frac{c_{0} / 4}{\log \log (q \tau)}$ then

$$
\left|L^{(k)}(s, \chi)\right| \ll \log \log (q \tau)^{k},\left|\frac{L^{\prime}}{L}(s, \chi)\right| \ll \log \log (q \tau) .
$$

Proof. Part $(i)$ is classical and and the proofs can be found in [9, pp. 331343]. For part $(i i)$ we shall follow the argument for bounding $\zeta(s)$ presented in [18] pages $158-160$. We put $\mathfrak{a}=(1-\chi(-1)) / 2$ and we suppose without loss of generality that $t>0$. Suppose that there exists $c_{0}$ such that if $\rho=\beta+i \gamma$ is a zero of $L(s, \chi)$ then

$$
\beta<1-\frac{c_{0}}{\log \log (q(|\gamma|+4))} .
$$

In fact we can thus deduce that $\min _{\rho}\left(\frac{1}{\rho}+\frac{1}{z-\rho}\right) \geq 0$ for $z=a+i b$ where $b \geq b_{0}$ is sufficiently large and $a \geq 1-\frac{c_{0} / 2}{\log \log q t}$ (This follows from the argument in [18, pp. 158-159]). We now let $s=\sigma+i t$ be a fixed complex number with $t$ sufficiently large and $\sigma \geq 1-\frac{c_{0} / 4}{\log \log q t}$. We put $s_{0}=1+\eta+i t$ with $\eta=\frac{c_{0} / 4}{\log \log q t}$. Suppose that $w$ is a complex number satisfying $|w| \leq 4 \eta$. The point $s_{0}+w=\sigma^{\prime}+i t^{\prime}$ satisfies $t^{\prime} \geq b_{0}$ and $\sigma^{\prime} \geq 1-\frac{c_{0} / 2}{\log \log q t}$ and thus

$$
\operatorname{Re}\left(\frac{1}{\rho}+\frac{1}{s_{0}+w-\rho}\right) \geq 0 .
$$

Consider the function

$$
F(w)=\frac{L^{\prime}\left(s_{0}, \chi\right)}{L\left(s_{0}, \chi\right)}-\frac{L^{\prime}\left(s_{0}+w, \chi\right)}{L\left(s_{0}+w, \chi\right)} .
$$


By the explicit formula for $\frac{L^{\prime}}{L}(s, \chi)$ (see chapter 14 of [4])

$$
\begin{aligned}
F(w) & =\frac{1}{s_{0}+w-1}-\frac{1}{s_{0}-1}+\frac{1}{2}\left(\frac{\Gamma^{\prime}}{\Gamma}\left(\frac{s_{0}+w}{2}+\frac{\mathfrak{a}}{2}\right)-\frac{\Gamma^{\prime}}{\Gamma}\left(\frac{s_{0}}{2}+\frac{\mathfrak{a}}{2}\right)\right) \\
& -\sum_{\rho}\left(\frac{1}{\rho}+\frac{1}{s_{0}+w-\rho}\right)+\sum_{\rho}\left(\frac{1}{\rho}+\frac{1}{s_{0}-\rho}\right) .
\end{aligned}
$$

By (42) and Stirling's formula it follows that

$$
\operatorname{Re}(F(w)) \leq A \log \log q \tau+\left|\operatorname{Re} \sum_{\rho}\left(\frac{1}{\rho}+\frac{1}{s_{0}-\rho}\right)\right| .
$$

Now the sum is $\ll \sum_{\rho} \frac{1}{\left|\rho \| s_{0}-\rho\right|}$ since $\operatorname{Re}\left(s_{0}\right) \ll 1$. Writing $\rho=\beta+i \gamma$ we divide the above sum into intervals. Note that $s_{0}=1+\eta+i t$ with $t>0$.

$$
\begin{aligned}
& I_{1}=[2 t, \infty), I_{2}=[t+h, 2 t], I_{3}=[t-h, t+h], I_{4}=[1, t-h], \\
& I_{5}=[-1,1], I_{6}=[-1,-t], I_{7}=(-\infty,-t) .
\end{aligned}
$$

Moreover, we set for $j=1, \ldots, 7 \sigma_{j}=\sum_{\gamma \in I_{j}}\left(|\rho|\left|s_{0}-\rho\right|\right)^{-1}$. Before proceeding we note that $|\rho|=\sqrt{\beta^{2}+\gamma^{2}} \geq \max (|\gamma|,|\beta|)$ and

$$
\left|s_{0}-\rho\right|=\sqrt{(1+\eta-\beta)^{2}+(\gamma-t)^{2}} \geq \max ((1+\eta-\beta),|\gamma-\tau|) .
$$

We define $N(t, \chi)$ to be the number of zeros of $L(s, \chi)$ in the box $-\frac{1}{2} \leq$ $\operatorname{Re}(s) \leq \frac{3}{2}$ and $|\operatorname{Im}(s)| \leq t$. We shall employ the well-known bound $N(t, \chi) \ll t \log (q(|t|+2))$. We have

$$
\begin{gathered}
\sigma_{1}=\sum_{\gamma \geq 2 \tau} \frac{1}{\gamma|t-\gamma|} \ll \sum_{\gamma \geq 2 t} \gamma^{-2} \ll \frac{\log q t}{t}, \\
\sigma_{2} \ll \sum_{t+h \leq \gamma \leq 2 t} \frac{1}{\gamma(\gamma-t)} \ll \int_{t+h}^{2 t} \frac{d N(u, \chi)}{u(u-t)} \ll \log (q t) \int_{t+h}^{2 t} \frac{d u}{u(u-t)} \ll \frac{\log (q t) \log (t / h)}{t}, \\
\sigma_{3}=\sum_{t-h \leq \gamma \leq t+h} \frac{1}{\gamma(1+\eta-\beta)} \ll \frac{\log \log q \tau}{c_{0}^{\prime}} \frac{N(t+h, \chi)-N(t-h, \chi)}{t} \\
\ll \frac{h \log (q t) \log \log q \tau}{t}, \\
\sigma_{4}=\sum_{1 \leq \gamma \leq t-h} \frac{1}{|\rho|\left|s_{0}-\rho\right|} \ll \sum_{1 \leq \gamma \leq t-h} \frac{1}{\gamma(t-\gamma)} \ll \int_{1^{-}}^{t-h} \frac{d N(t, \chi)}{t(t-h)} \\
\ll(\log q t) \int_{1}^{t-h} \frac{d u}{u(u-h)} \ll \frac{\log q t}{t-h} . \\
\sigma_{5}=\sum_{|\gamma| \leq 1} \frac{1}{|\rho|\left|s_{0}-\rho\right|} \ll t^{-1} \sum_{|\gamma| \leq 1}|\rho|^{-1} \ll t^{-1} \beta_{\min }^{-1} N(1, \chi),
\end{gathered}
$$

where $\beta_{\min }$ is the smallest positive real zero of $L(s, \chi)$. By the large zero-free region conjecture $\beta_{\text {min }}>\frac{c_{0}}{\log \log (4 q)}$ and thus

$$
\sigma_{5} \ll t^{-1} \log \log (q) \log (q) .
$$


Similarly,

$$
\begin{gathered}
\sigma_{6}=\sum_{-t \leq \gamma \leq-1} \frac{1}{|\rho|\left|s_{0}-\rho\right|} \ll \sum_{-t \leq \gamma \leq-1} \frac{1}{|\gamma||t-\gamma|} \ll t^{-1} \sum_{-t \leq \gamma \leq-1} \frac{1}{|\gamma|} \ll \frac{\log q t}{t}, \\
\sigma_{7}=\sum_{\gamma \leq-t} \frac{1}{|\rho|\left|s_{0}-\rho\right|} \ll \sum_{|\gamma| \geq t} \frac{1}{|\gamma|^{2}} \ll \frac{\log q t}{t} .
\end{gathered}
$$

Combining bounds and choosing $h=t / \log (q \tau)$ we derive

$$
\operatorname{Re}(F(w)) \ll A \log \log (q \tau)+\frac{h \log (q \tau) \log \log (q \tau)}{t} \ll \log \log (q \tau) .
$$

Since we have $\left|s-s_{0}\right| \leq 2 \eta$ it follows from the Borel-Caratheodory theorem that

$$
\left|\frac{L^{\prime}}{L}(s, \chi)\right| \ll \log \log (q \tau)+\left|\frac{\zeta^{\prime}}{\zeta}\left(s_{0}, \chi\right)\right| \ll \log \log (q \tau) .
$$

Therefore

$$
\log \left(\frac{L(s, \chi)}{L\left(s_{0}, \chi\right)}\right)=\int_{s_{0}}^{s} \frac{L^{\prime}}{L}(w, \chi) d w \ll\left|s-s_{0}\right| \log \log (q \tau) \ll 1 .
$$

Now note that $\left|\log L\left(s_{0}, \chi\right)\right| \leq \log \zeta(1+\eta)=\log _{3}(q \tau)+O(1)$ and it follows that $\log L(s, \chi) \ll \log _{3}(q \tau)$ for $\operatorname{Re}(s) \geq 1-\frac{c_{0}}{\log \log q \tau}$. Now writing $L(s, \chi)=\exp (\log L(s, \chi))$ we have we have $|L(s, \chi)| \leq \exp (|\log L(s, \chi)|) \leq$ $\exp \left(\log _{3}(q \tau)+O(1)\right) \ll \log \log (q \tau)$.

\section{Evaluation of $\mathcal{M}_{0}$ : Proof of Proposition 4.3}

With the previous lemmas in hand we are now set to evaluate $\mathcal{M}_{0}$.

Proof. In (19) $)$ we set $l=(m, k), m=l m_{1}$, and $k=l k_{1}$ to obtain

$$
\mathcal{M}_{0}=\sum_{l \leq M} \sum_{k_{1} \leq M / l} \frac{y_{l k_{1}}}{l k_{1}} \frac{\mu\left(k_{1}\right)}{\phi\left(k_{1}\right)} \sum_{\substack{l m_{1} \leq \frac{l k_{1} T}{2 \pi} \\\left(m_{1}, k_{1}\right)=1}} a\left(m_{1} l\right) .
$$

Rewriting $k_{1}$ as $k$

$$
\mathcal{M}_{0}=\sum_{l k \leq M} \frac{y_{l k} \mu(k)}{l k \phi(k)} S\left(\frac{k T}{2 \pi} ; l, k\right)
$$

where

$$
S\left(\frac{k T}{2 \pi} ; l, k\right)=\sum_{\substack{m \leq \frac{k T}{2 \pi} \\(m, k)=1}} a(m l) \text { and } a(r)=\sum_{\substack{u v=r \\ v \leq M}}(\Lambda * \log )(u) x_{v} .
$$

Note that by Lemma 6.1 we may decompose this as

$$
S\left(\frac{k T}{2 \pi} ; l, k\right)=\sum_{g h=l} \sum_{\substack{g v \leq M \\(v, k h)=1}} x_{g v} \sum_{\substack{u v \leq \frac{k T}{2 \pi} \\(u, k)=1}}(\Lambda * \log )(h u) .
$$


We have by Lemma 6.5 that

$$
\sum_{\substack{u \leq \frac{k T}{2 \pi v} \\(u, k)=1}}(\Lambda * \log )(h u)=\frac{T \phi(k)}{2 \pi v}\left(X_{1}(h, k, v)+X_{2}(h, k, v)\right)+O\left(\frac{\tau(h) k T}{v} \exp \left(-C_{2} \sqrt{\log T}\right)\right)
$$

since $\log \left(\frac{k T}{v}\right) \asymp \log T$ and

$$
\begin{aligned}
& X_{1}(h, k, v)=\frac{1}{2} \log \left(\frac{k T}{2 \pi v}\right)^{2}+2 \log \left(\frac{k T}{2 \pi v e}\right) \log (h)+(\Lambda * \log )(h), \\
& X_{2}(h, k, v)=\left(C_{0}-\eta_{1}(k)\right) \log \left(\frac{k T}{2 \pi v e}\right)+a_{1} \eta_{1}(k)-\eta_{2}(k)-g(h, k) .
\end{aligned}
$$

We set $\mathcal{M}_{0}=\mathcal{M}_{0}^{\prime}+\mathcal{M}_{0}^{\prime \prime}$ where $\mathcal{M}_{0}^{\prime}$ is the contribution in $\mathcal{M}_{0}$ arising from $X_{1}$ and $X_{2}$ in (44) and $\mathcal{M}_{0}^{\prime \prime}$ denote contribution arising from the the error term in (44). First the error term is

$$
\begin{aligned}
& \mathcal{M}_{0}^{\prime \prime} \ll T \exp \left(-C_{2} \sqrt{\log T}\right) \sum_{l k \leq M} \frac{\left|y_{l k}\right|}{l \phi(k)} \sum_{g h=l} \tau(h) \sum_{v \leq M / g} \frac{\left|x_{g v}\right|}{v} \\
& \ll T \exp \left(-C_{2} \sqrt{\log T}\right) \log \log M|| \frac{x_{n}}{n} \|_{1} \sum_{l k \leq M} \frac{(\tau *|x|)(l)\left|y_{l k}\right|}{l k} \\
& \ll T \exp \left(-C_{3} \sqrt{\log T}\right)|| \frac{x_{n}}{n}\left\|_{1}\right\| \frac{\left(\tau_{3} *|x|\right)(n) y_{n}}{n} \|_{1} .
\end{aligned}
$$

We now deal with $\mathcal{M}_{0}^{\prime}$ :

$$
\begin{aligned}
\mathcal{M}_{0}^{\prime} & =\frac{T}{2 \pi} \sum_{l k \leq M} \frac{y_{l k} \mu(k)}{l k} \sum_{g h=l} \sum_{\substack{g v \leq M \\
(v, k h)=1}} \frac{x_{g v}}{v}\left(X_{1}(h, k, v)+X_{2}(h, k, v)\right) \\
& =\frac{T}{2 \pi} \sum_{g h k \leq M} \frac{y_{g h k} \mu(k)}{g h k} \sum_{\substack{g v \leq M \\
(v, k h)=1}} \frac{x_{g v}}{v}\left(X_{1}(h, k, v)+X_{2}(h, k, v)\right) .
\end{aligned}
$$

By the variable change $h k=u$ we have

$$
\mathcal{M}_{0}^{\prime}=\frac{T}{2 \pi} \sum_{g u \leq M} \frac{y_{g u}}{g u} \sum_{\substack{g v \leq M \\(v, u)=1}} \frac{x_{g v}}{v} \sum_{h k=u} \mu(k)\left(X_{1}(h, k, v)+X_{2}(h, k, v)\right) .
$$

Next we will check that

$$
\sum_{h k=u} \mu(k) X_{1}(h, k, v)=-\frac{1}{2} \Lambda_{2}(u)+\log \left(\frac{T}{2 \pi v e}\right) \Lambda(u)+\frac{1}{2} \log \left(\frac{T}{2 \pi v}\right)^{2} \delta(u)
$$


This follows immediately from the identities:

$$
\begin{aligned}
& \sum_{d \mid u} \mu(d)=\delta(u):=\left\{\begin{array}{ll}
1 & \text { if } u=1 \\
0 & \text { if } u>1
\end{array},\right. \\
& \sum_{d \mid u} \mu(d) \log d=-\Lambda(u), \\
& \sum_{d \mid u} \mu(d)(\log d)^{2}=-2(\log u) \Lambda(u)+\Lambda_{2}(u), \\
& \sum_{d e=u} \mu(d)(\log d)(\log e)=(\log u) \Lambda(u)-\Lambda_{2}(u), \\
& \Lambda_{2}(u)=\Lambda(u) \log (u)+(\Lambda * \Lambda)(u) .
\end{aligned}
$$

Our next step is to compute $\sum_{h k=u} \mu(k) X_{2}(h, k, v)$. We recall that Lemma 6.2 gives us

$$
\begin{aligned}
\phi_{1}(u) & =\sum_{h k=u} \mu(k) \eta_{1}(k), \phi_{2}(u)=\sum_{h k=u} \mu(k) \eta_{2}(k), \\
\phi_{3}(u) & =\sum_{h k=u} \mu(k) g(h, k), \phi_{4}(u)=\sum_{h k=u} \mu(k) \eta_{1}(k) \log (k) .
\end{aligned}
$$

It follows from the definitions (30) and Lemma 6.2 that

$$
\begin{gathered}
\sum_{h k=u} \mu(k) X_{2}(h, k, v)=\log \left(\frac{T}{2 \pi v e}\right)\left(C_{0} \delta(u)-\phi_{1}(u)\right) \\
-C_{0} \Lambda(u)-\phi_{4}(u)+a_{1} \phi_{1}(u)-\phi_{2}(u)-\phi_{3}(u) .
\end{gathered}
$$

Combining these identities we arrive at

$$
\begin{aligned}
& \sum_{h k=u} \mu(k)\left(X_{1}(h, k, v)+X_{2}(h, k, v)\right)=-\frac{1}{2} \Lambda_{2}(u)+R_{1}\left(\log \left(\frac{T}{2 \pi v}\right)\right) \Lambda(u) \\
& \quad+\frac{1}{2} R_{2}\left(\log \left(\frac{T}{2 \pi v}\right)\right) \delta(u)+\alpha_{1}(u) \tilde{R}_{1}\left(\log \left(\frac{T}{2 \pi v}\right)\right)+\alpha_{2}(u)
\end{aligned}
$$

where $R_{1}, R_{2}, \tilde{R}_{1}$ are monic polynomials of degrees $1,2,1$. Note that $\alpha_{1}(u)=$ $-\phi_{1}(u)$ and $\alpha_{1}$ is supported on prime powers. In fact, $\alpha_{1}\left(p^{\alpha}\right)=\frac{\log p}{p-1}$. Also $\alpha_{2}(u)=a_{1} \phi_{1}(u)-\phi_{2}(u)-\phi_{3}(u)-\phi_{4}(u)$ and it is supported on those integers $n$ with $\omega(n) \leq 2$. Moreover, we have

$$
\begin{aligned}
\alpha_{2}\left(p^{\alpha}\right) & =-\frac{(\alpha+1)(\log p)^{2}}{p-1}-\frac{\log p}{p-1}\left(a_{1}+\frac{p}{p-1}\right) \\
\alpha_{2}\left(p^{\alpha} q^{\beta}\right) & =-(\log p)(\log q)\left(\frac{1}{p-1}+\frac{1}{q-1}\right) .
\end{aligned}
$$

Therefore

$$
\begin{aligned}
\mathcal{M}_{0}^{\prime}= & \frac{T}{2 \pi} \sum_{g u \leq M} \frac{y_{g u}}{g u} \sum_{\substack{g v \leq M \\
(v, u)=1}} \frac{x_{g v}}{v}\left(-\frac{1}{2} \Lambda_{2}(u)+R_{1}\left(\log \left(\frac{T}{2 \pi v}\right)\right) \Lambda(u)\right. \\
& \left.+\frac{1}{2} R_{2}\left(\log \left(\frac{T}{2 \pi v}\right)\right) \delta(u)+\tilde{R}_{1}\left(\log \left(\frac{T}{2 \pi v}\right)\right) \alpha_{1}(u)+\alpha_{2}(u)\right) .
\end{aligned}
$$


We define $H(M ; u, v)=\sum_{g \leq \min \left(\frac{M}{u}, \frac{M}{v}\right)} \frac{y_{u g} x_{v g}}{g}$ and thus

$$
\begin{aligned}
\mathcal{M}_{0} & =\frac{T}{2 \pi} \sum_{\substack{u, v \leq M \\
(u, v)=1}} \frac{c^{\prime}(u, v) H(M ; u, v)}{a v}+\frac{T}{4 \pi} \sum_{g v \leq M} \frac{y_{g} x_{g v}}{g v} R_{2}\left(\log \frac{T}{2 \pi v}\right) \\
& +O\left(T \exp \left(-C_{3} \sqrt{\log T}\right)\left\|\frac{x_{n}}{n}\right\|_{1}\left\|\frac{\left(\tau_{3} *|x|\right)(n) y_{n}}{n}\right\|_{1}\right)
\end{aligned}
$$

where

$$
c^{\prime}(u, v)=-\frac{1}{2} \Lambda_{2}(u)+R_{1}\left(\log \left(\frac{T}{2 \pi v}\right)\right) \Lambda(u)+\tilde{R}_{1}\left(\log \left(\frac{T}{2 \pi v}\right)\right) \alpha_{1}(u)+\alpha_{2}(u) .
$$

Now in the resonator case the error term $O\left(\frac{\tau(h) k T}{v} \exp \left(-C_{2} \sqrt{\log T}\right)\right)$ in (44) above is just replaced by $O\left(\frac{\tau(h) k T}{v} \exp \left(-\frac{C_{2}^{\prime} \log T}{\log \log T}\right)\right)$. The argument then proceeds identically and yields the same formula as above for $\mathcal{M}_{0}$ except with the error term

$$
O\left(T \exp \left(-\frac{C_{3}^{\prime} \log T}{\log \log T}\right)\left\|\frac{x_{n}}{n}\right\|_{1}\left\|\frac{\left(\tau_{3} *|x|\right)(n) y_{n}}{n}\right\|_{1}\right) .
$$

\section{Bounding $\mathcal{E}$ : Proof of Theorem 4.4}

In (20) we invert summation order and replace the variables $k$ by $k q$ and $m$ by $m d$ to obtain

$$
\begin{aligned}
\mathcal{E} & =\sum_{1<q \leq M} \sum_{\psi \bmod q}^{*} \tau(\bar{\psi}) \sum_{k \leq M / q} \frac{y_{k q}}{k q} \sum_{d \mid k q} \delta(q, k q, d, \psi) \sum_{m \leq \frac{k q T}{2 \pi d}} a(m d) \psi(m) \\
& =\sum_{k \leq M} \frac{\mathcal{N}\left(\frac{M}{k}, \frac{k T}{2 \pi}, k\right)}{k}
\end{aligned}
$$

where

$$
\mathcal{N}(\xi, z, k)=\sum_{2 \leq q \leq \xi} \frac{y_{k q}}{q} \sum_{\psi \bmod q}^{*} \tau(\bar{\psi}) \sum_{d \mid k q} \delta(q, k q, d, \psi) \sum_{m \leq q z / d} a(m d) \psi(m) .
$$

In our analysis of $\mathcal{N}(\xi, z, k)$ we have to distinguish between the two cases for the coefficients $x_{n}, y_{n}$. We define

$$
\eta= \begin{cases}\mathscr{L}^{A} & \text { in the divisor case } \\ \exp \left(\frac{2.5 \log T}{\log \log T}\right) & \text { in the resonator case }\end{cases}
$$

for an arbitrary positive constant $A>0$. We now estimate the sum $\mathcal{N}(\xi, z, k)$ by dividing up the range of $q$ into $2 \leq q \leq \eta$ and $\eta<q \leq \xi \leq M$. The case $2 \leq q \leq \eta$ is analogous to the Siegel-Walfisz theorem. That is, we shall estimate directly the sum $\sum_{m \leq q z / d} a(m d) \psi(m)$ by the classical contour integral method invoking the zero-free region for Dirichlet $L$-functions and Siegel's bound for the exceptional zero. The case $\eta<q \leq \xi$ is analogous to Gallagher [6] and Vaughan's [19] proofs of the Bombieri-Vinogradov theorem. Here we shall employ an analytic form of the large sieve inequality for 
Dirichlet characters. Thus we shall divide up $\mathcal{E}$ as:

$$
\begin{aligned}
\mathcal{E} & =\sum_{k<M / \eta} \frac{\mathcal{N}\left(\eta, \frac{k T}{2 \pi}, k\right)}{k}+\sum_{M / \eta<k \leq M} \frac{\mathcal{N}\left(\frac{M}{k}, \frac{k T}{2 \pi}, k\right)}{k} \\
& +\sum_{k \leq M / \eta} \frac{\mathcal{N}\left(\frac{M}{k}, \frac{k T}{2 \pi}, k\right)-\mathcal{N}\left(\eta, \frac{k T}{2 \pi}, k\right)}{k} .
\end{aligned}
$$

We abbreviate this to $\mathcal{E}=\mathcal{E}_{1}+\mathcal{E}_{2}+\mathcal{E}_{3}$. Shortly we shall establish

Proposition 8.1. ( $i$ ) If $x_{n}, y_{n}$ satisfy (2), (3) then

$$
\mathcal{E}_{1}+\mathcal{E}_{2} \ll T \exp \left(-C_{4} \sqrt{\log T}\right)\left\|\frac{j(k) \tau_{r}(k) \tau_{r+3}(k)}{k}\right\|_{1} \eta^{\frac{3}{2}+\epsilon}
$$

for some $C_{4}=C_{4}(A)>0$ where $\eta=\mathscr{L}^{A}$.

(ii) Assume the large zero-free region conjecture. If $x_{n}=y_{n}=f(n)$ then

$$
\mathcal{E}_{1}+\mathcal{E}_{2} \ll \exp \left(\frac{\left(-c_{0} / 8+o(1)\right) \log T}{\log \log T}\right)\left\|\frac{j(k) f(k)\left(\tau_{3} * f\right)(k)}{k}\right\|_{1} \eta^{\frac{3}{2}} .
$$

where $\eta=\exp \left(\frac{2.5 \log T}{\log \log T}\right)$.

We also show that

Proposition 8.2. (i) If $x_{n}, y_{n}$ satisfy (2), (3) then there exists a $C_{5}>0$ such that

$$
\mathcal{E}_{3} \ll T \mathscr{L}^{C_{5}}\left\|\frac{\tau_{r}(k)^{2}}{k}\right\|{ }_{1}^{2} \eta^{-1 / 2}+T^{\frac{3}{4}+\frac{\theta}{2}+\epsilon}
$$

where $\eta=\mathscr{L}^{A}$.

(ii) Assume the large zero-free region conjecture. If $x_{n}=y_{n}=f(n)$ then

$$
\mathcal{E}_{3} \ll T \mathscr{L}^{3}\left\|\frac{f(k)^{2}}{k}\right\|_{1}\left\|\frac{f(k)(\tau * f)(k)}{k}\right\|_{1} \eta^{-\frac{1}{2}}+\|f\|_{\infty}^{2} T^{\frac{3}{4}+\frac{\theta}{2}+\epsilon} .
$$

where $\eta=\exp \left(\frac{2.5 \log T}{\log \log T}\right)$.

With the above bounds for $\mathcal{E}_{1}, \mathcal{E}_{2}$, and $\mathcal{E}_{3}$ we deduce Theorem 4.4 which provides a bound for $\mathcal{E}$.

Proof of Theorem 4.4. We begin with part $(i)$ : the divisor case. Since $\eta=$ $\mathscr{L}^{A},\left\|\frac{\tau_{r}(k)^{2}}{k}\right\|_{1},\left\|\frac{j(k) \tau_{r}(k) \tau_{r+3}(k)}{k}\right\|_{1} \ll \mathscr{L}^{C^{\prime}}$ it follows from Propositions 8.1 and 8.2 that

$$
\mathcal{E} \ll T \exp \left(-C_{6} \sqrt{\log T}\right)+T L^{C_{7}-0.5 A}+T^{\frac{3}{4}+\frac{\theta}{2}+\epsilon}
$$

for some $C_{6}=C_{6}(A), C_{7}>0$. Choosing $A=2\left(A^{\prime}+C_{7}\right)$ yields

$$
\mathcal{E} \ll_{A^{\prime}} T(\log T)^{-A^{\prime}}+T^{\frac{3}{4}+\frac{\theta}{2}+\epsilon} .
$$

We next prove part $(i i)$ of the theorem: the resonator case. Since $\eta=$ $\exp \left(\frac{2.5 \mathscr{L}}{\log \mathscr{L}}\right)$,

$$
\left\|\frac{f(k)^{2}}{k}\right\|_{1},\left\|\frac{j(k) f(k)\left(\tau_{j} * f\right)(k)}{k}\right\|_{1} \ll \exp \left(\frac{(0.5+o(1)) \log T}{\log \log T}\right),
$$


and $\|f\|_{\infty} \ll T^{\theta}$ it follows that

$$
\mathcal{E} \ll T\left(\exp \left(\frac{\left(-c_{0} / 8+4.25+o(1)\right) \log T}{\log \log T}\right)+\exp \left(\frac{(-0.25+o(1)) \log T}{\log \log T}\right)\right)+T^{\frac{3}{4}+\frac{3 \theta}{2}+\epsilon}
$$

If $c_{0}$ is sufficiently large we have established there exists a $C_{8}>0$ such that $\mathcal{E} \ll T \exp \left(-\frac{C_{8} \log T}{\log \log T}\right)+T^{\frac{3}{4}+\frac{3 \theta}{2}+\epsilon}$. This completes the proof of Theorem 4.4. We have now reduced the proof to establishing the bounds of Propositions 8.1 and 8.2 .

8.1. Bounding $\mathcal{E}_{1}, \mathcal{E}_{2}$ : Proof of Proposition 8.1. In this section we will bound $\mathcal{E}_{1}$ and $\mathcal{E}_{2}$ and thus establish Proposition 8.1. Note that $\mathcal{E}_{1}$, $\mathcal{E}_{2}$ each take the form

$$
\mathcal{E}_{i}=\sum_{k \leq M} \frac{\mathcal{N}\left(\xi, \frac{k T}{2 \pi}, k\right)}{k} \quad(i=1,2)
$$

with $\xi \leq \eta$.

Proof of Proposition 8.1. We shall evaluate $\mathcal{N}\left(\xi, \frac{k T}{2 \pi}, k\right)$ by invoking the bound from Lemma 6.6 for $\delta(q, k, d, \psi)$ and the following:

Lemma 8.3. Let $\psi$ be a non-principal character modulo $q, T \ll w \ll T^{2}$, and $d \ll T$.

Divisor case. For any $A>0$ there exists a $C_{9}>0$ such that

$$
\sum_{m \leq w} a(m d) \psi(m) \ll_{A} j(d)(\tau *|x|)(d) w \exp \left(-C_{9} \sqrt{\log T}\right)
$$

for all $q \leq \mathscr{L}^{A}$.

Resonator case. Assume the large zero-free region conjecture for $L(s, \psi)$.

Then we have

$$
\sum_{m \leq w} a(m d) \psi(m) \ll j(d)(\tau *|x|)(d) w \exp \left(-\frac{\left(c_{0} / 8\right) \log T}{\log \log T}\right) .
$$

for $q \leq \exp \left(\frac{2.5 \mathscr{L}}{\log \mathscr{L}}\right)$.

The evaluation of $\mathcal{N}\left(\xi, \frac{k T}{2 \pi}, k\right)$ is split in the two cases.

We first consider part $(i)$. That is, $x_{n}, y_{n}$ satisfy (2) and (3). By Lemma 8.3 we obtain for $\xi \ll \eta=\mathscr{L}^{A}$

$$
\begin{aligned}
& \mathcal{N}\left(\xi, \frac{k T}{2 \pi}, k\right) \\
& \ll k T \exp \left(-C_{9} \mathscr{L}^{1 / 2}\right) \sum_{q \leq \xi_{k}}\left|y_{k q}\right| \sum_{\psi \bmod q}^{*}|\tau(\bar{\psi})| \sum_{d \mid k q}|\delta(q, k q, d, \psi)| \frac{j(d)(\tau * x)(d)}{d}
\end{aligned}
$$

By Lemma 6.6, $|\tau(\bar{\psi})| \leq \sqrt{q},\left|x_{n}\right|,\left|y_{n}\right| \ll \mathscr{L}^{C} \tau_{r}(n)$ we see that $\mathcal{N}\left(\xi, \frac{k T}{2 \pi}, k\right)$ is bounded by

$$
\ll \frac{j(k) \tau_{r}(k) k T}{\phi(k)} \exp \left(-C_{10} \sqrt{\mathscr{L}}\right) \sum_{q \leq \xi} j(q) \tau_{r}(q) \sqrt{q} \sum_{d \mid k q} \frac{(d, k)\left(\tau * \tau_{r}\right)(d)}{d} .
$$


By Lemma 6.7 and $\frac{k}{\phi(k)} \ll \log \mathscr{L}$ this is further bounded by

$$
\ll j(k) \tau_{r+3}(k) \tau_{r}(k) T \exp \left(-C_{11} \sqrt{\mathscr{L}}\right) \sum_{q \leq \eta} j(q) \tau_{r}(q) \sqrt{q} .
$$

The last sum is $\ll \eta^{\frac{3}{2}+\epsilon}$ and thus

$$
\mathcal{N}\left(\xi, \frac{k T}{2 \pi}, k\right) \ll j(k) \tau_{r+3}(k) \tau_{r}(k) T \exp \left(-C_{11} \sqrt{\mathscr{L}}\right) \eta^{\frac{3}{2}+\epsilon}
$$

for some $C_{11}>0$. Therefore by (57) we have

$$
\mathcal{E}_{1}+\mathcal{E}_{2} \ll T \exp \left(-C_{11} \sqrt{\log T}\right)\left\|\frac{j(k) \tau_{r+3}(k) \tau_{r}(k)}{k}\right\|_{1} \eta^{\frac{3}{2}+\epsilon} .
$$

We now establish part (ii). Here we assume $x_{n}=y_{n}=f(n)$ and $\eta=$ $\exp \left(\frac{2.5 \mathscr{L}}{\log \mathscr{L}}\right)$. As before $\mathcal{N}\left(\xi, \frac{k T}{2 \pi}, k\right)$ is bounded by

$$
k T \exp \left(-\frac{\left(c_{0} / 8\right) \mathscr{L}}{\log \mathscr{L}}\right) \sum_{q \leq \xi_{k}} f(k q) \sum_{\psi \bmod q}^{*}|\tau(\bar{\psi})| \sum_{d \mid k q}|\delta(q, k q, d, \psi)| \frac{j(d)(\tau * f)(d)}{d} .
$$

By Lemma 6.6 and $|\tau(\bar{\psi})| \leq \sqrt{q}$, we further bound this by

$$
\ll \frac{j(k) f(k) k T}{\phi(k)} \exp \left(-\frac{\left(c_{0} / 8\right) \mathscr{L}}{\log \mathscr{L}}\right) \sum_{q \leq \xi} j(q) f(q) \sqrt{q} \sum_{d \mid k q} \frac{(d, k)(\tau * f)(d)}{d} .
$$

By Lemma 6.7 we obtain

$$
\ll \frac{j(k)\left(\tau_{3} * f\right)(k) f(k) k T}{\phi(k)} \exp \left(-\frac{\left(c_{0} / 8\right) \mathscr{L}}{\log \mathscr{L}}+o(\sqrt{\mathscr{L}})\right) \sum_{q \leq \eta} j(q) f(q) \sqrt{q} .
$$

Obviously $\sum_{q \leq \eta} j(q) f(q) \sqrt{q} \ll \eta^{\frac{3}{2}}\left\|\frac{f(k)}{k}\right\|_{1}$. Thus

$$
\mathcal{N}\left(\xi, \frac{k T}{2 \pi}, k\right) \ll j(k)\left(\tau_{3} * f\right)(k) f(k) T \exp \left(\frac{\left(-\left(c_{0} / 8\right)+o(1)\right) \mathscr{L}}{\log \mathscr{L}}\right)\left\|\frac{f(k)}{k}\right\|_{1} \eta^{\frac{3}{2}} .
$$

Therefore by (57)

$$
\mathcal{E}_{1}+\mathcal{E}_{2} \ll T \exp \left(\frac{\left(-c_{0} / 8+o(1)\right) \mathscr{L}}{\log \mathscr{L}}\right)\left\|\frac{j(k)\left(\tau_{3} * f\right)(k) f(k)}{k}\right\|_{1}\|f(k) / k\|_{1} \eta^{\frac{3}{2}} .
$$

8.2. Proof of Lemma 8.3. We now establish Lemma 8.3 which was central to establishing our bounds for $\mathcal{E}_{1}, \mathcal{E}_{2}$.

Proof. Recall that $T \ll w \ll T^{2}$ and $d \ll T$. By Perron's formula, we have

$$
\sum_{m \leq w} a(m d) \psi(m)=\frac{1}{2 \pi i} \int_{\kappa-i U}^{\kappa+i U} A(s, \psi, d) w^{s} \frac{d s}{s}+O(\epsilon)
$$

where $\kappa=1+O\left((\log w)^{-1}\right)$ and

$$
\epsilon \ll \sum_{n \neq w}\left(\frac{w}{n}\right)^{\kappa}|a(d n)| \min \left(1, U^{-1}|\log (w / n)|^{-1}\right)+|a(w)| .
$$

We will first show that $\epsilon$ is small. Let $\epsilon=\epsilon_{1}+\epsilon_{2}$ where $\epsilon_{1}$ is the contribution from those terms with $n>1.5 \mathrm{w}$ and $n<0.5 \mathrm{w}$ and $\epsilon_{2}$ consists of the 
other terms. We observe that $(\Lambda * \log )(n) \leq \log ^{2} n$ and hence $|a(n)| \leq$ $\left(\log ^{2} n\right)(1 *|x|)(n)$. It follows that $\epsilon_{1}$ is bounded by

$\frac{w}{U} \sum_{n=1}^{\infty} \frac{|a(d n)|}{n^{\kappa}} \ll \frac{w}{U}(1 *|x|)(d)\left(\mathscr{L}^{2} \sum_{n \geq 1} \frac{(1 *|x|)(n)}{n^{\kappa}}+\sum_{n \geq 1} \frac{\log ^{2} n(1 *|x|)(n)}{n^{\kappa}}\right)$.

Observe that

$$
\sum_{n=1}^{\infty} \frac{(1 *|x|)(n)}{n^{\kappa}}=\sum_{a=1}^{\infty} \frac{1}{a^{\kappa}} \sum_{b \leq M} \frac{\left|x_{b}\right|}{b^{\kappa}} \ll \mathscr{L}\left\|x_{n} / n\right\|_{1} .
$$

A similar calculation gives

$$
\sum_{n=1}^{\infty} \frac{(\log n)^{2}(1 *|x|)(n)}{n^{\kappa}} \ll \mathscr{L}^{3}\left\|\frac{x_{n}}{n}\right\|_{1}
$$

and thus $\epsilon_{1} \ll \frac{w}{U} \mathscr{L}^{3}(1 *|x|)(d)\left\|\frac{x_{n}}{n}\right\|_{1}$. We now deal with $\epsilon_{2}$. Since $n d \ll T^{3}$ the contribution from those terms in $0.5 w \leq n<w$ is

$$
\begin{aligned}
& \sum_{0.5 w<n<w}|a(n d)| \min \left(1, U^{-1}|\log (w / n)|^{-1}\right) \\
& \ll(1 *|x|)(d) \mathscr{L}^{2} \sum_{0.5 w<n<w}(1 *|x|)(n) \min \left(1, U^{-1}|\log (w / n)|^{-1}\right) .
\end{aligned}
$$

Since $|\log (w / n)|^{-1} \ll \frac{n}{|w-n|}$ for $w / 2 \leq n<w$ it follows that the last sum is

$$
\sum_{w-\frac{w}{U} \leq n<w}(1 *|x|)(n)+\frac{w}{U} \sum_{\frac{w}{2}<n<w-\frac{w}{U}} \frac{(1 *|x|)(n)}{w-n} .
$$

However by Lemma 6.4 the first sum is $\ll U^{-1}\left\|\frac{x_{n}}{n}\right\|_{1}$. In the second sum we divide it up in to intervals of the form $\left[w-(k+1) \frac{w}{U}, w-k \frac{w}{U}\right]$ for $1 \leq k \leq K$ with $K \ll U$. By another application of Lemma 6.4 the second term is

$$
\begin{aligned}
& U^{-1} \sum_{k=1}^{K} \sum_{w-(k+1) \frac{w}{U}<n<w-k \frac{w}{U}} \frac{(1 *|x|)(n)}{w-n} \ll w^{-1} \sum_{k \leq K} \frac{1}{k} \sum_{w-(k+1) \frac{w}{U}<n<w-k \frac{w}{U}}(1 *|x|)(n) \\
& \ll w^{-1} \sum_{k \leq K} \frac{1}{k} \frac{w}{U}\left\|\frac{x_{n}}{n}\right\|_{1} \ll U^{-1} \mathscr{L} \|_{\frac{x_{n}}{n} \|_{1} .}
\end{aligned}
$$

Note that an identical argument applies to the range $w<n<1.5 w$ and thus $\epsilon_{2} \ll(1 *|x|)(d) U^{-1} \mathscr{L}\left\|\frac{x_{n}}{n}\right\|_{1}$. In summary,

$$
\sum_{m \leq w} a(m d) \psi(m)=\frac{1}{2 \pi i} \int_{\kappa-i U}^{\kappa+i U} A(s, \psi, d) w^{s} \frac{d s}{s}+O\left(\frac{(1 *|x|)(d)\left\|\frac{x_{n}}{n}\right\|_{1} w \mathscr{L}^{3}}{U}\right) \text {. }
$$

To complete the proof we require a bound for

$$
I:=\frac{1}{2 \pi i} \int_{\kappa-i U}^{\kappa+i U} A(s, \psi, d) w^{s} \frac{d s}{s} .
$$

In order to achieve this we need some understanding of the generating function $A(s, \psi, d)$. We will show that the generating function $A(s, \psi, d)$ can be computed explicitly in terms of $L(s, \psi)$. With our knowledge of $A(s, \psi, d)$ 
in hand we shall deform our contour left into the zero-free region of $L(s, \psi)$ and then bound $A(s, \psi, d)$ on this contour. Since $a(n)=(\Lambda * \log * x)(n)$, Lemma 6.1 yields

$$
A(s, \psi, d)=\sum_{d_{1} d_{2} d_{3}=d} A_{1}\left(s, 1, d_{1}\right) A_{2}\left(s, d_{1}, d_{2}\right) A_{3}\left(s, d_{1} d_{2}, d_{3}\right)
$$

where

$$
\begin{aligned}
& A_{1}(s, u, v)=\sum_{\substack{m v<M \\
(m, u)=1}} \frac{\psi(m) x_{m v}}{m^{s}}, \\
& A_{2}(s, u, v)=\sum_{(m, u)=1} \frac{\psi(m) \log (m v)}{m^{s}}, \\
& A_{3}(s, u, v)=\sum_{(m, u)=1} \frac{\psi(m) \Lambda(m v)}{m^{s}} .
\end{aligned}
$$

A calculation demonstrates that

$A_{2}(s, u, v)=L^{\prime}(s, \psi) \Phi(s, \psi, u)-L(s, \psi) \Phi^{\prime}(s, \psi, u)+(\log v) L(s, \psi) \Phi(s, \psi, u)$ where $\Phi(s, \psi, u)=\prod_{p \mid u}\left(1-\psi(p) p^{-s}\right)=\sum_{n \mid u} \frac{\mu(n) \psi(n)}{n^{s}}$ and

$$
A_{3}(s, u, v)= \begin{cases}-\frac{L^{\prime}}{L}(s, \chi)-\sum_{p \mid u} \frac{\chi(p) \log p}{p^{s}-\chi(p)} & \text { if } v=1 \\ \frac{\log p}{1-\chi(p) p^{-s}} & \text { if } v=p^{l},(u, p)=1 \\ \log p & \text { if } v=p^{l}, p \mid u \\ 0 & \text { else }\end{cases}
$$

With these expressions in hand we now analyze the behaviour of $A(s, \psi, d)$ to the right of the line $\operatorname{Re}(s)=1$. For $\operatorname{Re}(s) \geq 1 / 2,|\Phi(s, \psi, u)| \leq j(d)$, $\left|\Phi^{\prime}(s, \psi, u)\right| \ll j(d)(\log d)$ and thus

$$
\begin{gathered}
\left|A_{2}(s, u, v)\right| \ll j(d)\left(\left|L^{\prime}(s, \psi)\right|+|L(s, \psi)| \mathscr{L}\right), \\
\left|A_{3}(s, u, v)\right| \ll\left|\frac{L^{\prime}}{L}(s, \psi)\right|+\mathscr{L} .
\end{gathered}
$$

It follows from (59) and these two last bounds that

$$
|A(s, \psi, d)| \leq j(d)\left(\left|L^{\prime}(s, \psi)\right|+|L(s, \psi)| \mathscr{L}\right)\left(\left|\frac{L^{\prime}}{L}(s, \psi)\right|+\mathscr{L}\right) \sum_{d_{1} d_{2} d_{3}=d}\left|A\left(s, 1, d_{1}\right)\right|
$$

Now since $\left|A\left(s, 1, d_{1}\right)\right| \ll\left|x_{d_{1}}\right||| \frac{x_{n}}{n} \|_{1} M^{1-\sigma}$ and by the bounds for $L^{(j)}(s, \psi)$, $\frac{L^{\prime}}{L}(s, \psi)$ from Lemma 6.8 we obtain

$$
|A(s, \psi, d)| \leq j(d)(\tau *|x|)(d) \mathscr{L}^{3}\left\|\frac{x_{n}}{n}\right\|_{1} M^{1-\sigma}
$$

unconditionally for $\operatorname{Re}(s) \geq 1-\frac{c}{\log (q(|t|+4))}$ and assuming the large zero-free region conjecture it is true for $\operatorname{Re}(s) \geq 1-\frac{c_{0} / 4}{\log \log (q(|t|+4))}$. We are now prepared to bound $I$. The argument is again split in two cases.

Case 1. Divisor case. It follows that $A(s, \psi, d)$ has a meromorphic continuation to all of $\mathbb{C}$. For all non-principal characters, $A(s, \psi, d)$ has at most 
one simple pole in the region $\left\{s=\sigma+i t: \sigma \geq \sigma_{1}(t)=1-\frac{c}{\log q(|t|+2)}\right\}$ where $c>0$ is an absolute effective constant. By Siegel's theorem, this pole, if it exists is a real number $\beta$ that satisfies

$$
1-\beta \gg_{\epsilon} q^{-\epsilon}
$$

where the constant is ineffective. We shall let $\Gamma_{1}$ denote the contour $\sigma=$ $\sigma_{1}(U)$ and $|t| \leq U$. By (61) and $\left\|\frac{x_{n}}{n}\right\|_{1} \ll \mathscr{L}^{C^{\prime}}$ we have

$$
|A(s, \psi, d)| \ll j(d)(\tau *|x|)(d) \mathscr{L}^{C^{\prime \prime}}
$$

where $s=\sigma_{0}(U)+i t,|t| \leq U,|s-1| \gg \mathscr{L}^{-1}$, and $|s-\beta| \gg \mathscr{L}^{-1}$. It follows from Cauchy's theorem that

$$
\begin{aligned}
\sum_{m \leq w} a(m d) \psi(m) & \ll \int_{\Gamma_{1}}\left|A(s, \psi, d) \frac{w^{s}}{s}\right| d s \\
& +\left|\operatorname{res}_{s=\beta} A(s, \psi, d) \frac{w^{s}}{s}\right|+\frac{w}{U}(\tau *|x|)(d) \mathscr{L}^{4}|| \frac{x_{n}}{n} \|_{1} .
\end{aligned}
$$

By $(63)$

$$
\begin{aligned}
\int_{\Gamma_{1}} A(s, \psi, d) \frac{w^{s}}{s} d s & \ll j(d)(\tau *|x|)(d) \mathscr{L}^{C^{\prime \prime}} w \exp \left(\frac{-c \log w}{\log (q(U+2))}\right) \\
& \ll j(d)(\tau *|x|)(d) \mathscr{L}^{C^{\prime \prime}} w \exp \left(-C_{12} \sqrt{\log w}\right)
\end{aligned}
$$

since $q \leq \mathscr{L}^{A}$ and $U=\exp \left(C_{13} \sqrt{\log w}\right)$. To bound the residue at $s=\beta$ (the possible Siegel zero) we invoke Siegel's ineffective bound (62) to obtain

$$
w^{\beta} \leq w \exp \left(-\frac{C_{13} \log w}{q^{\epsilon}}\right) \leq w \exp \left(-\frac{C_{13} \log w}{\mathscr{L}^{\epsilon A}}\right) \ll w \exp \left(-C_{14} \sqrt{\mathscr{L}}\right)
$$

if $\epsilon \leq(2 A)^{-1}$. Thus

$$
\operatorname{res}_{s=\beta} A(\beta, \psi, d) \frac{w^{s}}{s} \ll j(d)(\tau * x)(d) \mathscr{L}^{C^{\prime \prime}} w \exp \left(-C_{14} \sqrt{\mathscr{L}}\right) .
$$

Collecting all estimates yields

$$
\sum_{m \leq w} a(m d) \psi(m) \ll_{A} j(d)(\tau *|x|)(d) w \exp \left(-C_{15} \sqrt{\mathscr{L}}\right) .
$$

where $C_{15}=C_{15}(A)>0$ and $q \leq \mathscr{L}^{A}$.

Case 2. Resonator case. In this case we assume that for each $q$ that every primitive Dirichlet $L$-function $L(s, \psi)$ is non-vanishing in the region $\left\{s=\sigma+i t: \sigma \geq \sigma_{2}(t)=1-\frac{c_{0} / 4}{\log \log (q(|t|+4))}\right\}$. We shall let $\Gamma_{2}$ denote the contour with $\sigma=\sigma_{2}(U)$ and $|t| \leq U$. By (61) and Lemma 6.8

$$
|A(s, \psi, d)| \ll j(d)(\tau *|x|)(d) \mathscr{L}^{4}|| \frac{x_{n}}{n} \|_{1} \exp \left(\frac{c_{0} / 4 \log M}{\log \log M}\right)
$$

where $s=\sigma_{2}(U)+i t,|t| \leq U,|s-1| \gg \mathscr{L}^{-1}$. By Cauchy's theorem

$$
\sum_{m \leq w} a(m d) \psi(m) \ll \int_{\Gamma_{2}}\left|A(s, \psi, d) \frac{w^{s}}{s}\right| d s+\frac{w}{U}(\tau *|x|)(d) \mathscr{L}^{4}\left\|\frac{x_{n}}{n}\right\|_{1} .
$$


Hence

$$
\int_{\Gamma_{2}} A(s, \psi, d) \frac{w^{s}}{s} d s \ll j(d)(\tau *|x|)(d) \mathscr{L}^{5}\left\|\frac{x_{n}}{n}\right\|_{1} w \exp \left(\frac{c_{0}}{4}\left(\frac{\log M}{\log _{2} M}-\frac{\log w}{\log _{2}(q(U+2))}\right)\right)
$$

We choose $U=\exp \left(\frac{C_{17} \log w}{\log \log w}\right)$. Since $q \leq \exp \left(\frac{2.5 \mathscr{L}}{\log \mathscr{L}}\right), T \ll w \ll T^{2}$ we have

$$
\int_{\Gamma_{2}} A(s, \psi, d) \frac{w^{s}}{s} d s \ll j(d)(\tau *|x|)(d)\left\|\frac{x_{n}}{n}\right\|_{1} w \exp \left(\frac{c_{0}}{4}(\theta-1)(1+o(1)) \frac{\mathscr{L}}{\log \mathscr{L}}\right)
$$

As $\left\|\frac{x_{n}}{n}\right\|_{1} \ll \exp (o(\sqrt{\mathscr{L}}))$ and $0<\theta<\frac{1}{2}$ we deduce

$$
\sum_{m \leq w} a(m d) \psi(m) \ll j(d)(\tau *|x|)(d) w \exp \left(-\frac{\left(c_{0} / 8\right) \log T}{\log \log T}\right)
$$

for $q \leq \exp \left(\frac{2.5 \mathscr{L}}{\log \mathscr{L}}\right)$

8.3. Bounding $\mathcal{E}_{3}$ : Proof of Proposition 8.2 , We now prove the bound for

$$
\mathcal{E}_{3}=\sum_{k \leq M / \eta} \frac{\mathcal{N}\left(\frac{M}{k}, \frac{k T}{2 \pi}, k\right)-\mathcal{N}\left(\eta, \frac{k T}{2 \pi}, k\right)}{k}
$$

Proof of Proposition 8.2. By Perron's formula applied with $U=T^{20}, T \ll$ $w \ll T^{2}$, and $\kappa=1+(\log w)^{-1}$ we have

$$
\sum_{m \leq w} a(m d) \psi(m)=\frac{1}{2 \pi i} \int_{\kappa-i U}^{\kappa+i U} A(s, \psi, d) w^{s} \frac{d s}{s}+O\left(w^{\epsilon}\right) .
$$

Combining this expression with the definition (51) of $\mathcal{N}$ we obtain

$$
\begin{aligned}
& \left|\mathcal{N}\left(\frac{M}{k}, \frac{k T}{2 \pi}, k\right)-\mathcal{N}\left(\eta, \frac{k T}{2 \pi}, k\right)\right| \ll \sum_{\eta \leq q \leq \frac{M}{k}} \frac{\left|y_{k q}\right|}{q} \\
& \cdot \sum_{\psi \bmod q}^{*}|\tau(\bar{\psi})| \sum_{d \mid k q}|\delta(q, k q, d, \psi)|\left(\left|\int_{\kappa-i U}^{\kappa+i U} A(s, \psi, d)\left(\frac{q k T}{2 \pi d}\right)^{s} \frac{d s}{s}\right|+O\left(T^{\epsilon}\right)\right)
\end{aligned}
$$

By Lemma 6.6 the term containing $O\left(T^{\epsilon}\right)$ contributes

$$
\ll T^{\epsilon} \sum_{\eta \leq q \leq \frac{M}{k}} \frac{\left|y_{k}\right|\left|y_{q}\right|}{\sqrt{q}} \sum_{\psi \bmod q}^{*} \sum_{d \mid k q} \frac{(d, k)}{\phi(k) \phi(q)} \ll T^{\epsilon} \sum_{\eta \leq q \leq \frac{M}{k}} \frac{\left|y_{k}\right|\left|y_{q}\right| \sigma_{1}(k q)}{\phi(k) \sqrt{q}} \ll\left|y_{k}\right| T^{\epsilon} M^{\frac{3}{2}} .
$$

The first sum in (67) is bounded by

$$
\begin{aligned}
& \ll \frac{\left|y_{k}\right|(\log \mathscr{L})}{\phi(k)} \sum_{\eta \leq q \leq \frac{M}{k}} \frac{\left|y_{q}\right| \sqrt{q}}{q \phi(q)} \sum_{\psi \bmod q}^{*} \sum_{d \mid k q} \mu^{2}(k q)(d, k)\left|\int_{\kappa-i U}^{\kappa+i U} A(s, \psi, d)\left(\frac{q k T}{2 \pi d}\right)^{s} \frac{d s}{s}\right| \\
& \ll \frac{\left|y_{k}\right|(\log \mathscr{L})}{\phi(k)} \max _{Q \leq \frac{M}{k}}\left(\mu^{2}(k Q) \sum_{d \mid k Q}(d, k) \int_{\eta}^{\frac{M}{k}} z^{-1} d S(z)\right)
\end{aligned}
$$


where

$$
S(z)=\sum_{q \leq z} \frac{q^{1 / 2}\left|y_{q}\right|}{\phi(q)} \sum_{\psi \bmod q}^{*}\left|\int_{\kappa-i U}^{\kappa+i U} A(s, \psi, d)\left(\frac{q k T}{2 \pi d}\right)^{s} \frac{d s}{s}\right| .
$$

The next step is to dissect $A(s, \psi, d)=\sum_{m=1}^{\infty} a(m d) \psi(m) m^{-s}$ via Vaughan's identity. We define the partial sum of $A(s, \psi, d)$

$$
F=F(s, \psi, d)=\sum_{m \leq u} \frac{a(m d) \psi(m)}{m^{s}}
$$

and the partial sum $G(s, \psi)$ of $L(s, \psi)^{-1}$ by

$$
G=G(s, \psi)=\sum_{m \leq v} \frac{\mu(m) \psi(m)}{m^{s}} .
$$

We choose the parameters

$$
u=z^{2} \text { and } v=T^{1 / 2}
$$

where $z$ is a real variable satisfying $\eta \leq z \leq \frac{M}{k}$. Vaughan's identity is

$$
A=(A-F)(1-L G)+(F-F L G+A L G) \text {. }
$$

We write this as $A=H+I$ where

$$
H=(A-F)(1-L G) \text { and } I=F-F L G+A L G .
$$

It follows that

$$
\frac{1}{2 \pi i} \int_{\kappa-i U}^{\kappa+i U} A(s, \psi, d)\left(\frac{q k T}{2 \pi d}\right)^{s} \frac{d s}{s}=\frac{1}{2 \pi i} \int_{\kappa-i U}^{\kappa+i U}(H+I)(s, \psi, d)\left(\frac{q k T}{2 \pi d}\right)^{s} \frac{d s}{s} .
$$

By the argument of [3. p. 514] we have

$$
\int_{\kappa-i U}^{\kappa+i U} I(s, \psi, d)\left(\frac{q k T}{2 \pi d}\right)^{s} \frac{d s}{s}=\int_{\frac{1}{2}-i U}^{\frac{1}{2}+i U} I(s, \psi, d)\left(\frac{q k T}{2 \pi d}\right)^{s} \frac{d s}{s}+O\left(T^{-1}\right) .
$$

Next we define

$$
\mathcal{H}(z)=\sum_{q \leq z} \frac{\left|y_{q}\right| q^{3 / 2}}{\phi(q)} \sum_{\psi \bmod q}^{*} \int_{-U}^{U}|H(\kappa+i t)| \frac{d t}{\kappa+|t|}
$$

where $\kappa=1+O\left(\mathscr{L}^{-1}\right)$ and

$$
\mathcal{I}(z)=\sum_{q \leq z} \frac{\left|y_{q}\right| q}{\phi(q)} \sum_{\psi \bmod q}^{*} \int_{-U}^{U}|I(1 / 2+i t)| \frac{d t}{1 / 2+|t|}
$$

With these definitions in hand we obtain

$$
\left|\mathcal{N}\left(\frac{M}{k}, \frac{k T}{2 \pi}, k\right)-\mathcal{N}\left(\eta, \frac{k T}{2 \pi}, k\right)\right| \ll \sigma_{1}+\sigma_{2}+\left|y_{k}\right| M^{\frac{3}{2}} T^{\epsilon}
$$


where

$$
\begin{aligned}
& \sigma_{1}=\frac{(T k)\left|y_{k}\right| \log \mathscr{L}}{\phi(k)} \max _{Q \leq \frac{M}{k}} \mu^{2}(k Q) \sum_{d \mid k Q} \frac{(d, k)}{d} \int_{\eta}^{\frac{M}{k}} z^{-1} d \mathcal{H}(z), \\
& \sigma_{2}=\frac{(T k)^{1 / 2}\left|y_{k}\right| \log \mathscr{L}}{\phi(k)} \max _{Q \leq \frac{M}{k}} \mu^{2}(k Q) \sum_{d \mid k Q} \frac{(d, k)}{d^{1 / 2}} \int_{\eta}^{\frac{M}{k}} z^{-1} d \mathcal{I}(z) .
\end{aligned}
$$

Next we will show the bounds

$$
\begin{gathered}
\int_{\eta}^{\frac{M}{k}} z^{-1} d \mathcal{H}(z) \ll(1 *|x|)(d) \mathscr{L}^{5}\left\|\frac{x_{k}^{2}}{k}\right\|_{1}^{1 / 2}\left\|\frac{x_{k}}{k}\right\|_{1}\left(\eta^{-1 / 2}+T^{-\delta} k^{-1 / 2}\right) \\
\int_{\eta}^{\frac{M}{k}} z^{-1} d \mathcal{I}(z) \ll j(d) \tau_{3}(d)|x(d)|^{2}\left(T^{\theta+\epsilon} k^{-\frac{1}{2}}+T^{\frac{\theta}{2}+\frac{1}{4}+\epsilon}\right)
\end{gathered}
$$

where $\delta=\frac{1}{4}-\frac{\theta}{2}$. We deduce

$$
\sigma_{1} \ll T \mathscr{L}^{7}\left\|\frac{x_{k}^{2}}{k}\right\|_{1}^{1 / 2}\left\|\frac{x_{k}}{k}\right\|_{1}\left(\eta^{-1 / 2}+T^{-\delta} k^{-1 / 2}\right)\left|y_{k}\right| \max _{Q \leq \frac{M}{k}} \mu^{2}(k Q) \sum_{d \mid k Q} \frac{(d, k)(1 *|x|)(d)}{d},
$$

$$
\sigma_{2} \ll \frac{\left|y_{k}\right|}{k^{\frac{1}{2}}} \max _{Q \leq \frac{M}{k}} \mu^{2}(k Q) \sum_{d \mid k Q} \frac{(d, k) j(d) \tau_{3}(d)|x(d)|^{2}}{d^{1 / 2}}\left(T^{\frac{1}{2}+\theta+\epsilon} k^{-\frac{1}{2}}+T^{\frac{3}{4}+\frac{\theta}{2}+\epsilon}\right) .
$$

We now bound $\left|\mathcal{N}\left(\frac{M}{k}, \frac{k T}{2 \pi}, k\right)-\mathcal{N}\left(\eta, \frac{k T}{2 \pi}, k\right)\right|$ in the two cases:

Case 1. Divisor case. We have by Lemma 6.7 (i)

$$
\begin{aligned}
\sigma_{1} & \ll T \mathscr{L}^{C_{18}}\left(\eta^{-1 / 2}+T^{-\delta} k^{-1 / 2}\right) \tau_{r}(k) \max _{Q \leq \frac{M}{k}} \mu^{2}(k Q) \sum_{d \mid k Q} \frac{(d, k) \tau_{r+1}(d)}{d} \\
& \ll T \mathscr{L}^{C_{19}}\left(\eta^{-1 / 2}+T^{-\delta} k^{-1 / 2}\right) \tau_{r}(k) \tau_{r+2}(k) .
\end{aligned}
$$

By Lemma $6.7(i)$ and $\left|x_{n}\right|,\left|y_{n}\right| \ll T^{\epsilon}$ we obtain

$$
\sigma_{2} \ll T^{\frac{1}{2}+\theta+\epsilon} k^{-1 / 2}+T^{\frac{3}{4}+\frac{\theta}{2}+\epsilon} .
$$

From (66), (68) and our bounds for $\sigma_{i}$ we have

$$
\begin{aligned}
\mathcal{E}_{3} & \ll T \mathscr{L}^{C_{19}} \sum_{k \leq M / \eta} \frac{\tau_{r}(k) \tau_{r+2}(k)}{k}\left(\eta^{-1 / 2}+T^{-\delta} k^{-1 / 2}\right)+T^{\frac{1}{2}+\theta+\epsilon}+T^{\frac{3}{4}+\frac{\theta}{2}+\epsilon} \\
& \ll T \mathscr{L}^{C_{20}} \eta^{-\frac{1}{2}}+T^{\frac{3}{4}+\frac{\theta}{2}+\epsilon}
\end{aligned}
$$

for $0<\theta<1 / 2$ as claimed.

Case 2. Resonator case. By (69) and (8) we have

$\sigma_{1} \ll T \exp \left(\frac{(0.25+o(1)) \mathscr{L}}{\log \mathscr{L}}\right)\left(\eta^{-1 / 2}+T^{-\delta} k^{-1 / 2}\right) f(k) \max _{Q \leq \frac{M}{k}} \mu^{2}(k Q) \sum_{d \mid k Q} \frac{(d, k)(1 * f)(d)}{d}$ 
By an application of Lemma 6.7 (ii) this is

$$
\sigma_{1} \ll T \exp \left(\frac{(0.25+o(1)) \mathscr{L}}{\log \mathscr{L}}\right)\left(\eta^{-1 / 2}+T^{-\delta} k^{-1 / 2}\right) f(k)(\tau * f)(k) .
$$

By (72), Lemma 6.7 $(i i)$ and $f(d),(\tau * f)(d) \ll\|f\|_{\infty} T^{\epsilon}$ (see (77)) we find

$$
\sigma_{2} \ll f(k)\|f\|_{\infty}^{2}\left(T^{\frac{1}{2}+\theta+\epsilon} k^{-1 / 2}+T^{\frac{3}{4}+\frac{\theta}{2}+\epsilon}\right)
$$

By (66), (68) and our expressions for the $\sigma_{i}$

$$
\mathcal{E}_{3} \ll T \exp \left(\frac{(0.25+o(1)) \mathscr{L}}{\log \mathscr{L}}\right)\left\|\frac{f(k)(\tau * f)(k)}{k}\right\|_{1} \eta^{-1 / 2}+\|f\|_{\infty}^{2}\left(T^{\frac{1}{2}+\theta+\epsilon}+T^{\frac{3}{4}+\frac{\theta}{2}+\epsilon}\right)
$$

since $\left\|\frac{f(k)}{k^{3 / 2}}\right\|_{1} \ll 1$ and $\left\|\frac{f(k)}{k}\right\|_{1} \ll M^{\epsilon}$.

\section{Establishing (69)}

The argument of Proposition 8.2 has been reduced to establishing (69) and (70). In this section we establish (69). We require the large sieve inequality:

$$
\sum_{q \leq z} \frac{q}{\phi(q)} \sum_{\psi \bmod q}^{*} \int_{-U}^{U}\left|\sum_{n} a_{n} \psi(n) n^{-i t}\right|^{2} \frac{d t}{\kappa+|t|} \ll \sum_{n}\left(n+z^{2}(\log U)\right)\left|a_{n}\right|^{2} .
$$

In addition, we define for an arbitrary function $\phi(s, \psi)$ the operator

$$
\mathcal{A}(\phi)=\sum_{q \leq z} \frac{q}{\phi(q)} \sum_{\psi \bmod q}^{*} \int_{-U}^{U}|\phi(1 / 2+i t, \psi)| \frac{d t}{\kappa+|t|} .
$$

Notice that if $c$ is a constant then $\mathcal{A}(c \phi)=|c| \mathcal{A}(\phi)$ and also for two functions $\phi_{i}=\phi_{i}(s, \psi)$ for $i=1,2$ we have $\mathcal{A}\left(\phi_{1} \phi_{2}\right) \leq \mathcal{A}\left(\phi_{1}^{2}\right)^{1 / 2} \mathcal{A}\left(\phi_{2}^{2}\right)^{1 / 2}$. Recall that $H=(A-F)(1-L G)$. By (73)

$$
\begin{aligned}
\mathcal{A}\left((A-F)^{2}\right) & =\sum_{q \leq z} \frac{q}{\phi(q)} \sum_{\psi \bmod q}^{*}|A(\kappa+i t)-F(\kappa+i t)|^{2} \frac{d t}{\kappa+|t|} \\
& \ll \sum_{m \geq u}\left(m+z^{2} \mathscr{L}\right)|a(m d)|^{2} m^{-2 \kappa} .
\end{aligned}
$$

Since $|a(m d)| \ll\left(\log ^{2} m\right) \mathscr{L}^{2}(1 *|x|)(d)(1 *|x|)(m)$ for $d \leq T$

$$
\mathcal{A}\left((A-F)^{2}\right) \ll(1 *|x|)^{2}(d) \mathscr{L}^{4} \cdot \sum_{m \geq u} \frac{\left(\log ^{4} m\right)(1 *|x|)^{2}(m)}{m^{2 \kappa}}\left(m+z^{2} \mathscr{L}\right) .
$$

Since $2 \kappa-1=1+O\left(\mathscr{L}^{-1}\right)$ we have

$$
\begin{aligned}
& \sum_{m \geq u} \frac{\left(\log ^{4} m\right)(1 *|x|)^{2}(m)}{m^{2 \kappa-1}}=\sum_{v_{1}, v_{2} \leq M}\left|x_{v_{1}}\right|\left|x_{v_{2}}\right| \sum_{\substack{m \geq u \\
\left[v_{1}, v_{2}\right] \mid m}} \frac{\log ^{4} m}{m^{2 \kappa-1}} \\
& =\sum_{v_{1}, v_{2} \leq M} \frac{\left|x_{v_{1}}\right|\left|x_{v_{2}}\right|}{\left[v_{1}, v_{2}\right]^{2 \kappa-1}} \sum_{m^{\prime} \geq \frac{u}{\left[v_{1}, v_{2}\right]}} \frac{\log ^{4}\left(m^{\prime}\left(v_{1}, v_{2}\right)\right)}{\left(m^{\prime}\right)^{2 \kappa-1}} \ll \mathscr{L}^{5} \sum_{v_{1}, v_{2} \leq M} \frac{\left|x_{v_{1}}\right|\left|x_{v_{2}}\right|}{\left[v_{1}, v_{2}\right]}
\end{aligned}
$$


Since $\left[v_{1}, v_{2}\right]^{-1}=\left(v_{1} v_{2}\right)^{-1} \sum_{g \mid\left(v_{1}, v_{2}\right)} \phi(g)$ the last expression is

$$
\ll \mathscr{L}^{5} \sum_{g \leq M} \frac{\phi(g)\left|x_{g}\right|^{2}}{g^{2}}\left(\sum_{\substack{v \leq \frac{M}{g} \\(v, g)=1}} \frac{\left|x_{v}\right|}{v}\right)^{2} \ll \mathscr{L}^{5}\left\|\frac{x_{n}^{2}}{n}\right\|_{1}\left\|\frac{x_{n}}{n}\right\|_{1}^{2} .
$$

An analogous calculation establishes

$$
\sum_{m>u} \frac{(\log m)^{4}(1 *|x|)(m)^{2}}{m^{2 \kappa}} \ll \mathscr{L}^{4}\left\|\frac{(1 *|x|)^{2}(k)}{k}\right\|_{1} u^{-1} \ll \mathscr{L}^{5}\left\|\frac{x_{n}^{2}}{n}\right\|_{1}\left\|\frac{x_{n}}{n}\right\|_{1}^{2} u^{-1} .
$$

Therefore $\mathcal{A}\left((A-F)^{2}\right) \ll(1 *|x|)(d)^{2} \mathscr{L}^{6}\left\|\frac{x_{n}^{2}}{n}\right\|_{1}\left\|\frac{x_{n}}{n}\right\|_{1}^{2}$. Moreover, in [3] it is established that $\mathcal{A}\left((1-L G)^{2}\right) \ll\left(1+z^{2} v^{-1}\right) \mathscr{L}^{4}$. Thus we obtain

$$
\begin{aligned}
\mathcal{H}(z) & \leq \sqrt{z} \mathcal{A}(|(A-F)(1-L G)|) \\
& \ll(1 *|x|)(d) \mathscr{L}^{5}\left\|\frac{x_{k}^{2}}{k}\right\|_{1}^{1 / 2}\left\|\frac{x_{k}}{k}\right\| \sqrt{z}\left(1+z T^{-1 / 4}\right)
\end{aligned}
$$

where in the last line we applied Cauchy-Schwarz. Since $M=T^{\theta}$ we deduce

$$
\int_{\eta}^{\frac{M}{k}} z^{-1} d \mathcal{H}(z) \ll(1 *|x|)(d) \mathscr{L}^{5}\left\|\frac{x_{k}^{2}}{k}\right\|_{1}^{1 / 2}\left\|\frac{x_{k}}{k}\right\|_{1}\left(\eta^{-1 / 2}+T^{\frac{\theta}{2}-\frac{1}{4}} k^{-1 / 2}\right) .
$$

\section{Establishing (70)}

In this section, we are not so precise about bounds. This is since we will have a small power savings from the main term. We set $s=1 / 2+i$ and we now provide a bound for $\mathcal{I}(z)$. Since $\mathcal{I}(z)=\mathcal{A}(F-F L G+A L G)$ we see that

(75) $\mathcal{I}(z) \ll \mathcal{A}\left(F^{2}\right)^{1 / 2} \mathcal{A}(1)^{1 / 2}+\mathcal{A}\left(F^{2}\right)^{1 / 2} \mathcal{A}\left(L^{4}\right)^{1 / 4} \mathcal{A}\left(G^{4}\right)^{1 / 4}+\mathcal{A}(|L A||G|)$.

It follows from (60) that

$$
|A(s, \psi, d) L(s, \psi)| \leq j(d) \tau_{3}(d) T^{\epsilon}\left(|L(s, \psi)|^{2}+\left|L^{\prime}(s, \psi)\right|^{2}\right)|\mathcal{B}(s)|
$$

for some Dirichlet polynomial $\mathcal{B}(s)=\sum_{m \leq y} \frac{b_{m}}{m^{s}}$ where $\left|b_{m}\right| \ll\left|x_{d}\right|\left|x_{m}\right|$. Thus

$$
\begin{aligned}
& \left.\mathcal{A}(|L A||G|) \leq j(d) \tau_{3}(d) T^{\epsilon} \mathcal{A}\left(|L(s, \psi)|^{2}+\left|L^{\prime}(s, \psi)\right|^{2}\right)|\mathcal{B}(s)||G(s, \psi)|\right) \\
& \ll j(d) \tau_{3}(d) T^{\epsilon} \mathcal{A}\left(|L(s, \psi)|^{4}+\left|L^{\prime}(s, \psi)\right|^{4}\right)^{1 / 2} \mathcal{A}\left(|\mathcal{B}(s, \psi)|^{4}\right)^{1 / 4} \mathcal{A}\left(|G(s, \psi)|^{4}\right)^{1 / 4}
\end{aligned}
$$

It suffices to bound $\mathcal{A}(\phi)$ for a variety of $\phi=\phi(s, \psi)$. We have the following bounds:

$$
\mathcal{A}(1), \mathcal{A}\left(|L(s, \psi)|^{4}\right), \mathcal{A}\left(\left|L^{\prime}(s, \psi)\right|^{4}\right) \ll z^{2} T^{\epsilon} .
$$

The first bound is trivial and the last two are due to an argument of Montgomery [8]. Next we analyze $\mathcal{A}\left(F^{2}\right), \mathcal{A}\left(G^{4}\right)$, and $\mathcal{A}\left(\mathcal{B}^{4}\right)$. Note that

$$
F(s, \psi)=\sum_{k \leq u} \frac{a(k d) \psi(k)}{k^{s}}, G(s, \psi)^{2}=\sum_{k \leq v^{2}} \frac{\beta_{k}}{k^{s}}, \mathcal{B}(s)^{2}=\sum_{k \leq M^{2}} \gamma_{k} k^{-s}
$$

where the coefficients satisfy

$$
a(k d) \ll T^{\epsilon}(\tau * x)(d)(\tau * x)(k),\left|\beta_{k}\right| \leq \tau(k),\left|\gamma_{k}\right| \ll x(d)^{2}(|x| *|x|)(k) .
$$


The large sieve (73) inequality yields

$$
\begin{aligned}
\mathcal{A}\left(F(s, \psi)^{2}\right) & \ll T^{\epsilon}(\tau *|x|)(d)^{2} \sum_{k \leq u}\left(k+z^{2} \mathscr{L}\right) \frac{(\tau *|x|)(k)^{2}}{k} \\
& \ll(\tau *|x|)(d)^{2} T^{\epsilon}\left(u+x^{2}\right) \\
\mathcal{A}\left(G(s, \psi)^{4}\right) & \ll \sum_{k \leq v^{2}}\left(k+z^{2} \mathscr{L}\right) \frac{\tau(k)^{2}}{k} \ll T^{\epsilon}\left(v^{2}+z^{2}\right), \\
\mathcal{A}\left(\mathcal{B}(s)^{4}\right) & \ll x(d)^{4} \sum_{k \leq M^{2}}\left(k+z^{2} \mathscr{L}\right) \frac{(|x| *|x|)^{2}(k)}{k} \leq T^{\epsilon} x(d)^{4}\left(M^{2}+z^{2}\right) .
\end{aligned}
$$

By (75), (76) , (177) and the bound $(a+b)^{1 / n} \ll a^{1 / n}+b^{1 / n}$ for $a, b>0$, $n \in \mathbb{N}$ we have

$$
\begin{aligned}
\mathcal{I}(x) & \ll(\tau *|x|)(d) z\left(u^{1 / 2}+z\right) T^{\epsilon} \\
& +(\tau *|x|)(d) z^{1 / 2}\left(u^{1 / 2}+z\right)\left(v^{1 / 2}+z^{1 / 2}\right) T^{\epsilon} \\
& +j(d) \tau_{3}(d)|x(d)| z\left(v^{1 / 2}+z^{1 / 2}\right)\left(M^{1 / 2}+z^{1 / 2}\right) T^{\epsilon} .
\end{aligned}
$$

Recalling that $u=z^{2}$ and $v=T^{1 / 4}$ this simplifies to

$$
\mathcal{I}(z) \ll j(d) \tau_{3}(d)|x(d)| T^{\epsilon}\left(z^{2}+z^{3 / 2} T^{1 / 4}+z^{3 / 2} M^{1 / 2}+z T^{1 / 4} M^{1 / 2}\right) .
$$

Since $M=T^{\theta}$

$$
\begin{aligned}
\int_{\eta}^{\frac{M}{k}} z^{-1} d \mathcal{I}(z) & \ll j(d) \tau_{3}(d)|x(d)| T^{\epsilon}\left(\frac{M}{k}+\left(\frac{M}{k}\right)^{1 / 2} T^{1 / 4}+\left(\frac{M}{k}\right)^{1 / 2} M^{1 / 2}+T^{1 / 4} M^{1 / 2}\right) \\
& \ll j(d) \tau_{3}(d)|x(d)|\left(T^{\theta+\epsilon} k^{-1 / 2}+T^{\frac{\theta}{2}+\frac{1}{4}+\epsilon}\right) .
\end{aligned}
$$

\section{REFERENCES}

[1] J.B. Conrey, A. Ghosh, S.M. Gonek. Large gaps between zeros of the zeta-function . Mathematika 33 (1986), no. 2, 212-238.

[2] J.B. Conrey, A. Ghosh, S.M. Gonek. Simple zeros of zeta functions. Colloque de Théorie Analytique des Nombres "Jean Coquet" (Marseille, 1985), 77-83, Publ. Math. Orsay, 88-02, Univ. Paris XI, Orsay, 1988.

[3] J.B. Conrey, A. Ghosh, and S.M. Gonek, Simple zeros of the Riemann zeta function, Proc. London Math. Soc. (3), 76 (1998), 497-522.

[4] H. Davenport, Multiplicative number theory, 3rd edn (revised by H.L. Montgomery), Graduate texts in mathematics 74, (Springer, New York, 1980).

[5] Akio Fujii, On a conjecture of Shanks, Proc. Japan Acad. Ser. A Math. Sci. 70 (1994), no. 4, 109-114.

[6] P.X. Gallagher, Bombieri's mean value theorem, Mathematika, 15 (1968), 1-6.

[7] S.M. Gonek, Mean values of the Riemann zeta function and its derivatives, Invent. Math. 75 (1984), 123-141.

[8] H. L. Montgomery, Topics in multiplicative number theory, Lecture Notes in Mathematics 227 (Springer, Berlin, 1971).

[9] Hugh L. Montgomery and Robert C. Vaughan, Multiplicative Number Theory: 1. Classical Theory, Cambridge University Press, Cambridge, 2006.

[10] Nathan Ng, The fourth moment of $\zeta^{\prime}(\rho)$, Duke Mathematical Journal, 125 no. 2 (2004), 243-266. 
[11] Nathan $\mathrm{Ng}$, The distribution of the summatory function of the Möbius function, Proc. London Math. Soc. (3) 89, 361-369.

[12] Nathan Ng, Large gaps between the zeros of the Riemann zeta function, submitted, http://www.mathstat. uottawa.ca/ ${ }^{\sim}$ nng362/RESEARCH/research.html.

[13] Nathan Ng, Extreme values of $\zeta^{\prime}(\rho), \quad$ submitted, http://www. mathstat. uottawa.ca/ ${ }^{\sim n n g} 362 / \mathrm{RESEARCH} / \mathrm{research} . \mathrm{html}$.

[14] Z. Rudnick, K. Soundararajan, Lower bounds for moments of $L$-functions. Proc. Natl. Acad. Sci. USA 102 (2005), no. 19, 6837-6838.

[15] Z. Rudnick, K. Soundararajan, Lower bounds for moments of $L$-functions: symplectic and orthogonal examples, Multiple Dirichlet Series, Automorphic Forms, and Analytic Number Theory (Editors: Friedberg, Bump, Goldfeld, and Hoffstein), Proc. Symp. Pure Math., vol. 75, Amer. Math. Soc., 2006.

[16] P. Shiu, A Brun-Titchmarsh theorem for multiplicative functions, J. Reine Angew. Math. 313 (1980), 161-170.

[17] K. Soundararajan. Extreme values of L-functions at the central point, preprint.

[18] G. Tenenbaum, Introduction to analytic and probabilistic number theory, Cambridge University Press, Cambridge, 1995.

[19] R.C. Vaughan, Mean value theorems in prime number theory, J. London Math. Soc. (2), 10 (1975), 153-162.

Nathan $\mathrm{Ng}$

Department of Mathematics and Statistics

University of Ottawa

585 King Edward Ave.

Ottawa, ON

Canada K1N6N5

nng@uottawa.ca 